\title{
Theoretical and Numerical Analysis of Void Coalescence in Porous Ductile Solids under Arbitrary Loadings
}

\author{
M. E. Torki ${ }^{1}$, C. Tekog̃lu ${ }^{2}$, J.-B. Leblond ${ }^{3}$, A. A. Benzerga ${ }^{1,4}$ \\ ${ }^{1}$ Department of Aerospace Engineering, Texas A\&M University, College Station, TX 77843, USA \\ ${ }^{2}$ Department of Mechanical Engineering, TOBB University of Economics and Technology, \\ Sög̃ütözü, Ankara, 06560, Turkey \\ ${ }^{3}$ Sorbonne Universites, UPMC Univ Paris 06, CNRS, UMR 7190 \\ Institut Jean Le Rond d'Alembert, F-75005, Paris, France \\ ${ }^{4}$ Department of Materials Science and Engineering, Texas A\&M University, College Station, TX 77843, USA
}

\begin{abstract}
Micromechanics-based constitutive relations are developed to model plasticity in solids with relatively high levels of porosity. They are especially appropriate to model void coalescence in ductile materials. The model is obtained by limit analysis of a cylindrical cell containing a coaxial void of finite height with plastic flow confined to the ligaments, and loaded under combined tension and shear. Previously obtained analytical estimates were not upper-bound preserving when shear was present and, in addition, were assessed against numerical results obtained for different cell geometries. Here, a rigorous upper-bound model is developed and its predictions are consistently compared with finite-element based estimates of limit loads on the same cylindrical unit cell exploiting quasi-periodic boundary conditions. The numerical results are used to guide a heuristic modification of the model in order to capture the behavior for extremely flat or extremely elongated voids.
\end{abstract}

Key Words: Ductile fracture; Low triaxiality; Internal necking; Internal shearing; Homogenization; Upper-bound.

\section{Introduction}

Void coalescence is known to be the last elementary stage of ductile failure (Pineau et al., 2016). That is, as soon as the first few largest voids approach each other within a fraction $(\sim 0.3-0.5)$ of the intervoid distance, yet long before they link up, the stress carrying capacity abruptly drops, and this upheaval continues to failure at the material point level (Koplik and Needleman, 1988, Benzerga, 2002). This sudden change is associated with strain concentration in the intervoid ligament (a form of micro-scale strain localization). Prior to this, void deformation occurs by diffuse plasticity, the distorsion being due to void enlargement, change of shape, rotation or all (Benzerga and Leblond, 2010, Benzerga et al., 2016). Ultimate failure of a test piece can thus occur if plastic flow successively localizes in intervoid ligaments thereby leading to macroscopic ductile crack growth. This mechanism prevails unless failure occurs by some plastic instability at the scale of many-void populations.

As a precursor to void coalescence, the process of micro-scale strain concentration should thus be modeled for predicting ductile fracture. This involves developing constitutive relations for voided solids in a 
"coalescence state", to be further defined below. When put together with available models for voided solids in "pre-coalescence states", e.g., (Gurson, 1977) the transition between the two states will correspond to the abrupt change in deformation mechanism.

It is noted that the developed constitutive relations are relevant to describe the plastic response of materials with relatively high levels of porosity, as for example considered by Fritzen et al. (2012). However, the resulting models are different from pre-coalescence, Gurson-like models, because of fundamental differences in the boundary conditions assumed in developing the said constitutive relations. What is of particular importance is that the porosity levels of interest can be quite low by comparison with those prevailing in engineered porous materials. Typical figures would be on the order of 0.01 , perhaps even smaller.

It is also emphasized that this type of models is different from those where void coalescence is viewed as an instability that can be predicted in terms of the pre-coalescence constitutive relations, e.g. (Danas and Ponte Castañeda, 2012); also see Benzerga et al. (2016). The fundamental premise of the line of models to be developed here is that the pre-coalescence constitutive relations cease to be valid at the critical point, and thus provide no basis for predicting localization; as explained by Rice (1976), "An alternative hypothesis would be that some essentially new physical deformation mechanism comes into play, abruptly, and rapidly degrades the strength of the material. In such cases the pre-localization constitutive relations cannot be continued analytically at the critical point, and they provide no basis for prediction of localization."

Internal necking of the intervoid ligaments, as the most prevalent mechanism for void coalescence (Pineau et al., 2016), has been inferred from the pioneering computational work of Koplik and Needleman (1988), approximately modeled by Thomason (1985), Benzerga et al. (1999), Pardoen and Hutchinson (2000), Gologanu et al. (2001) and Benzerga (2002), and recently tackled on more rigorous grounds by Benzerga and Leblond (2014) and Morin et al. (2015). A "coalescence state" is defined in this context as any state after a neck has initiated in the intervoid ligaments, with the deformation mode shifting to purely uniaxial, and elastic unloading taking place outside of these ligaments.

The void coalescence models listed above strictly apply to predominately tensile loadings even if approximate extensions have been attempted so as to incorporate more general loadings (Benzerga, 2002, Benzerga et al., 2004). In recent years, there has been revived interest in ductile fracture under combined tension and shear e.g. (Barsoum and Faleskog, 2007a, Dunand and Mohr, 2011). More generally, one needs to consider not only the normal stress acting on the localization plane but also the shear stress. When available and fully developed, such models can be utilized to analyze ductile fracture under more general loading schemes, as investigated in some recent works using micromechanical cell analyses (Leblond and Mottet, 2008, Barsoum and Faleskog, 2011, Scheyvaerts et al., 2011, Tvergaard, 2012, Nielsen et al., 2012, Dunand and Mohr, 2014, Tekog̃lu, 2014, Liu et al., 2016). The motivation in accounting for a shear component in the remote loading is two-fold. First, it is of interest to quantify how the internal necking condition is affected by the shear stress. Second, if loading is shear-dominated a seamless transition from internal necking to "internal shearing" may occur, that is with all shear deformation taken up within the intervoid ligament.

Any constitutive relations for a porous material involve dilatant plasticity, at least for associative plastic flow as envisaged here. The chief concern of this paper is to develop such relations for a porous material in which voids are in a state of incipient coalescence (by internal necking or shearing) and consider the effects of normal as well as shear stresses. It is assumed that the presence of a remote shear stress would not change the basic mechanism of strain concentration. There have been quite a few modeling efforts in this direction. Leblond and Mottet (2008) and later Tekog̃lu et al. (2012) developed such equations using a "sandwich model" whereby the void is smeared out in the central layer of the sandwich. Later, Torki et al. (2015) developed a closed-form solution to the problem of void coalescence under combined tension and shear by considering explicit expressions of the velocity field around the void. Recently, Keralavarma and Chockalingam (2016) extended the analysis of Benzerga and Leblond (2014) to plastically anisotropic matrices by considering a sub-family of velocity fields introduced by Morin et al. (2015). Their analysis also accounted for combined tension and shear loadings. However, the limit analysis procedures in both Torki 
et al. (2015) and Keralavarma and Chockalingam (2016) involved "uncontrolled" approximations which did not preserve the upper-bound character of the approach; see Benzerga and Leblond (2010) for background. In addition, Torki et al. (2015) offered some comparisons of their model predictions with the finite element results of Tekog̃lu et al. (2012). The model was derived on the basis of a cylindrical cell containing a cylindrical void whereas the calculations in Tekog̃lu et al. (2012) were for a tetragonal cell with a spheroidal void. Notable discrepancies were observed between the finite element results and the model predictions.

These discrepancies could be attributed a priori to three possible causes: (i) the choice of trial velocity fields in the analytical approach; (ii) the uncontrolled approximation made in the analytical approach; (iii) the difference between the geometries of the elementary cells considered in the analytical and numerical approaches. The aim of the present paper is to examine possible causes (ii) and (iii). To this end we develop an improved model preserving the rigorous upper-bound character and carry out cell model calculations using exactly the same cell geometry as that considered in the analytical model.

The paper is organized as follows. Section 2 is devoted to the derivation of the upper-bound model, with details deferred to Appendices A and B. Section 3 presents the principle of the finite element calculations that use the same cylindrical cell, with details about boundary conditions gathered in Appendix C. Finally, Section 4 reports our results, showing comparisons between the upper-bound and approximate models as well as between the new model and unit cell calculations.

\section{Analytical Model}

\subsection{GEOMETRY AND LOADING}

(a)

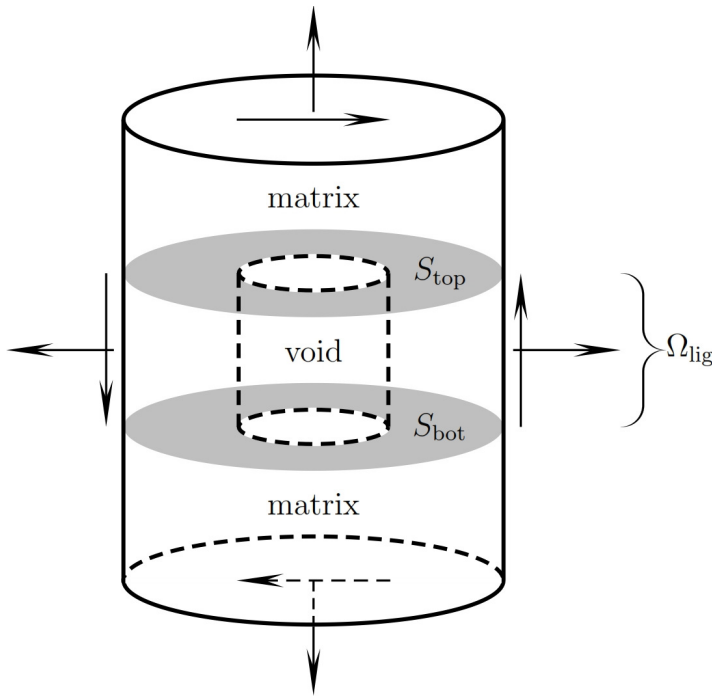

(b)

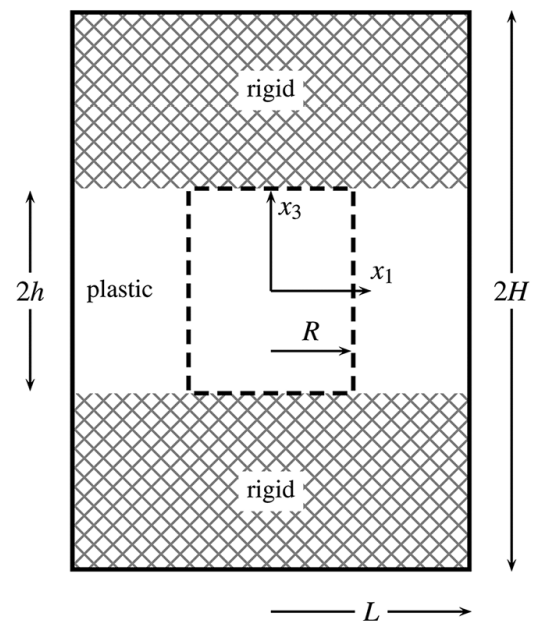

Figure 1: (a) Geometry of a cylindrical cell under combined tension and shear. (b) Cell parameters.

As in (Torki et al., 2015), the elementary volume $\Omega$ is a cylindrical cell of height $2 H$ and radius $L$ containing a coaxial cylindrical void $\omega$ of height $2 h$ and radius $R$, Fig. 1 . Use is made of both a local cylindrical basis $\left(\mathbf{e}_{r}, \mathbf{e}_{\theta}, \mathbf{e}_{z}\right)$ and a global Cartesian one $\left(\mathbf{e}_{1}, \mathbf{e}_{2}, \mathbf{e}_{3}\right)$. The displacement boundary conditions imposed on the unit cell lead to a macroscopic stress state with a predominant axial stress, $\Sigma_{33}>\Sigma_{11}, \Sigma_{33}>\Sigma_{22}$, as well as shear stresses, $\Sigma_{12}$ and $\Sigma_{31}$. With no loss of generality, the base vector $\mathbf{e}_{1}$ is taken parallel to the applied 
shear force. While not space filling, the analyzed geometry stands as a reasonable approximation of a unit cell in a periodic medium.

To represent "coalescence states" the cell is further divided into a central porous layer that defines the ligament domain, $\Omega_{\text {lig }}$, and fully dense regions above and below it. The interfaces between these two regions and the ligament are denoted $S_{\text {top }}$ and $S_{\text {bot }}$ (Fig. 1a) and their union $S_{\text {int }}$. The height of the ligament domain is set by the void height $2 h$; see Torki et al. (2015) for background. This geometry is uniquely determined by the independent dimensionless parameters identified in the first row of equation (1), respectively termed the void aspect ratio, the ligament parameter, and the cell aspect ratio.

$$
\begin{aligned}
W & =\frac{h}{R}, \quad \chi=\frac{R}{L} \quad, \quad \lambda=\frac{H}{L} \\
f_{\mathrm{b}} & \equiv \frac{\omega}{\Omega_{\text {lig }}}=\chi^{2} \\
c & \equiv \frac{\Omega_{\text {lig }}}{\Omega}=\frac{h}{H}=\frac{W \chi}{\lambda} \\
f & \equiv \frac{\omega}{\Omega}=c f_{\mathrm{b}}
\end{aligned}
$$

For convenience, Eq. (1) also introduces other auxiliary parameters which will be used in the derivations: $f_{\mathrm{b}}$ is the porosity within the ligament band, $c$ is the volume fraction of the band, and $f$ is the overall porosity.

\subsection{Structure of Constitutive Relations}

To mimic coalescence states, the regions above and below $\Omega_{\text {lig }}$ are modeled as rigid (Fig. 1b). In actual evolution problems using the cell model (Koplik and Needleman, 1988, Barsoum and Faleskog, 2007b, Dunand and Mohr, 2014), these regions would correspond to elastically unloaded ones. The mechanism of void growth abruptly changes due to the unloading that occurs above and below the cavity. Hence, plastic flow is assumed to be confined to the ligament and obey the von Mises yield criterion with the associated flow rule:

$$
\begin{aligned}
& \phi(\boldsymbol{\sigma})=\sigma_{\mathrm{eq}}^{2}-\bar{\sigma}^{2}=0 \quad \sigma_{\mathrm{eq}} \equiv \sqrt{\frac{3}{2} \sigma_{i j}^{\prime} \sigma_{i j}^{\prime}} \\
& d_{i j}=\frac{3}{2} \frac{d_{\mathrm{eq}}}{\bar{\sigma}} \sigma_{i j}^{\prime} \quad d_{\mathrm{eq}} \equiv \sqrt{\frac{2}{3} d_{i j} d_{i j}}
\end{aligned}
$$

where $\sigma^{\prime}$ is the stress deviator, and $\sigma_{\text {eq }}$ and $d_{\text {eq }}$ denote the von Mises equivalent stress and equivalent strain rate, respectively. Also, $\bar{\sigma}$ is the yield stress in simple tension.

The fundamental inequality of limit analysis gives rise to a variational definition of the effective yield criterion of a porous material as follows:

$$
\forall \mathbf{D}, \quad \boldsymbol{\Sigma}: \mathbf{D} \leq \Pi(\mathbf{D}), \quad \Pi(\mathbf{D})=\inf _{\mathbf{v} \in \mathcal{K}(\mathbf{D})}\left\langle\sup _{\sigma^{*} \in \mathcal{C}} \sigma_{i j}^{*} d_{i j}\right\rangle_{\Omega}
$$

if the velocity field is discontinuous across an interface $S$ then a surface term must be added to $\Pi(\mathbf{D})$ as

$$
\frac{1}{\Omega} \int_{S} \sup _{\sigma^{*} \in \mathcal{C}} t_{i}^{*} \llbracket v_{i} \rrbracket \mathrm{d} S
$$

In (3) $\boldsymbol{\Sigma}$ and $\mathbf{D}$ denote the effective stress and strain rate tensors, defined as volume averages of their microscopic counterparts $\boldsymbol{\sigma}$ and $\mathbf{d}$, and $\Pi(\mathbf{D})$ is the effective plastic dissipation. Also, $\langle\cdot\rangle_{\Omega}$ stands for averaging over $\Omega, \mathcal{K}(\mathbf{D})$ is the set of kinematically admissible velocity fields $\mathbf{v}$ compatible with $\mathbf{D}$, inf and sup, respectively, represent the infimum and supremum over a set, and $\mathcal{C}$ is the microscopic reversibility 
domain, the boundary of which is the yield surface of the matrix, here given by (2) 1 . In (4) $\mathbf{t}^{*}$ denotes the traction acting on the interface and $\llbracket \mathbf{v} \rrbracket$ the velocity jump across it.

Thus, stress states that lie within the effective reversibility domain, here denoted $\mathscr{C}$, are given by (3), possibly augmented by (4), and the effective yield surface is the boundary of that domain, $\partial \mathscr{C}$. The reader is referred to Benzerga and Leblond (2010) and Torki et al. (2015) for further details. When the effective dissipation function is differentiable the yield surface is smooth. In such cases, the yield surface is defined by the parametric equation:

$$
\Sigma_{i j}=\frac{\partial \Pi}{\partial D_{i j}}(\mathbf{D})
$$

where $\mathbf{D}$ is no longer arbitrary as in (3) 1 but represents the rate of deformation corresponding to $\boldsymbol{\Sigma}$ through the macroscopic flow rule.

\subsection{TRIAL VELOCITY FIELDS}

For general loadings, the exact velocity fields that minimize the integrals in $(3)_{2}$ cannot be obtained analytically. Nevertheless, use of trial ones leads to an upper bound to the yield surface (Benzerga and Leblond, 2010). Here, the trial velocity fields are taken from Torki et al. (2015) and are briefly recalled for completeness. On account of the existing rigid zones above and below the central void, the unit cell considered cannot deform along the $x_{1}$ and $x_{2}$ directions nor can it shear in the $x_{1}-x_{2}$ plane, i.e., $D_{11}=D_{22}=D_{12}=0$. In addition, the velocity jump across $S_{\text {int }}$, if any, must be purely tangential. Thus, the velocity ought to be consistent with the following constraints:

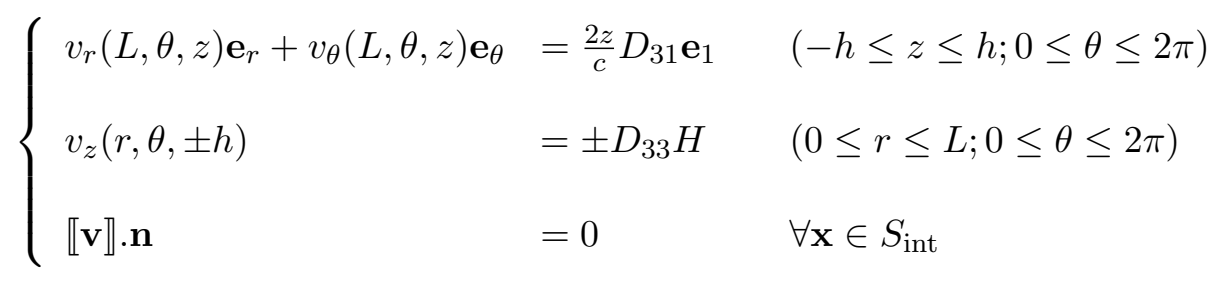

where $D_{31}$ and $D_{33}$ are the prescribed shear and axial strain rates, and $\mathbf{n}$ is the normal vector to the interface. Condition (6) 1 is supplemented by a constant velocity in the rigid zones $(h \leq|z| \leq H)$. Boundary conditions (6) stand as an appproximation of periodic boundary conditions and are consistent with the coalescence states defined above as in cell model studies (Koplik and Needleman, 1988, Barsoum and Faleskog, 2007b, Dunand and Mohr, 2014). It should be noted that owing to the presence of rigid zones in the cell, the velocity field cannot be consistent with uniform strain-rate boundary conditions (of the Hill-Mandel kind).

Details aside, the simplest trial velocity field that fulfills (6) along with the incompressibility condition ( $\operatorname{tr} \mathbf{d}=\operatorname{div} \mathbf{v}=0$ ) is given by (in the ligaments only):

$$
\mathbf{v}(\mathbf{x})=\left(\frac{A}{r}-B \frac{r}{2}\right) \mathbf{e}_{r}+B z \mathbf{e}_{z}+\frac{2 z}{c} D_{31} \mathbf{e}_{1}
$$

where $c$ is defined in (1) 3 , and parameters $A$ and $B$ are determined by boundary conditions as follows:

$$
A=\frac{D_{33} L^{2}}{2 c} \quad, \quad B=\frac{D_{33}}{c}
$$

The corresponding components of the microscopic rate of deformation tensor $\mathbf{d}$ were reported in Torki et al. (2015). Because of its relative simplicity, velocity field (7) is not continuous across $S_{\text {int }}$. 


\subsection{EFFECTIVE DisSIPATION}

Following Torki et al. (2015) an upper bound of the effective dissipation may be expressed as:

$$
\Pi=\Pi^{\mathrm{vol}}+\Pi^{\mathrm{surf}}
$$

with

$$
\left\{\begin{array}{l}
\Pi^{\text {vol }}=c\left(1-\chi^{2}\right)\left\langle\bar{\sigma} d_{\mathrm{eq}}\right\rangle_{\Omega_{\mathrm{lig}}-\omega} \\
\Pi^{\text {surf }}=\frac{1}{\Omega} \int_{S_{\mathrm{int}}} \frac{\bar{\sigma}}{\sqrt{3}}\left|\llbracket v_{\mathrm{t}} \rrbracket\right| \mathrm{d} S
\end{array}\right.
$$

where the surface term $\Pi^{\text {surf }}$ emerges as a result of the discontinuity of tangential velocity $\llbracket v_{t} \rrbracket$ and is thus a direct consequence of (4). The calculation of $\Pi^{\text {surf }}$ was carried out by Benzerga and Leblond (2014) to yield:

$$
\Pi^{\text {surf }}=\left|D_{33}\right| \Sigma^{\text {surf }}, \quad \Sigma^{\text {surf }}(\chi, W)=\frac{\bar{\sigma}}{3 \sqrt{3}} \frac{\chi^{3}-3 \chi+2}{\chi W}
$$

It is in the calculation of the volumetric term $\Pi^{\mathrm{vol}}$ that the present work differs from Torki et al. (2015). Indeed, to simplify their treatment Torki et al. made an approximation in evaluating (9) $)_{1}$ which did not preserve the upper-bound character. Here, careful treatment of this term is developed. In accordance with the calculations reported by Torki et al. (2015), the expression of $d_{\text {eq }}$ as stated in (2) reads

$$
d_{\mathrm{eq}}^{2}=\frac{D_{33}^{2}}{3 c^{2}}\left(3+\frac{L^{4}}{r^{4}}\right)+\frac{4 D_{31}^{2}}{3 c^{2}}
$$

Then the volumetric portion of the effective dissipation $\Pi^{\mathrm{vol}}$ of $(9)_{1}$ becomes:

$$
\Pi^{\mathrm{vol}} \equiv \Pi^{\mathrm{vol}}\left(D_{33}, D_{31}\right)=\left(1-\chi^{2}\right) \frac{\bar{\sigma}}{\sqrt{3}}\left\langle\sqrt{D_{33}^{2}\left(3+\frac{L^{4}}{r^{4}}\right)+4 D_{31}^{2}}\right\rangle_{\Omega_{\mathrm{lig}}-\omega}
$$

where the parameter $c$ has canceled out. The following approximation was exploited in Torki et al. (2015) to reach a simplified volumetric average in (12):

$$
\Pi^{\mathrm{vol}} \approx\left(1-\chi^{2}\right) \frac{\bar{\sigma}}{\sqrt{3}} \sqrt{\left\langle D_{33}^{2} \sqrt{\left(3+\frac{L^{4}}{r^{4}}\right)}\right\rangle_{\Omega_{\mathrm{lig}-\omega}}^{2}+4 D_{31}^{2}}
$$

However, this approximation, which was initially introduced by Tekog̃lu et al. (2012), destroys the rigorous upper-bound character, and therefore warrants some assessment against numerical estimates.

Unlike in (Torki et al., 2015, Tekog̃lu et al., 2012), no approximation is introduced herein. Introducing the change of variable $\left(D_{33}, D_{31}\right) \rightarrow\left(D_{33}, \bar{D}\right)$ with

$$
\bar{D}^{2}=3 D_{33}^{2}+4 D_{31}^{2}
$$

with the constraint $\bar{D} D_{33} \geq 0, \Pi^{\mathrm{vol}}$ can be written more concisely as

$$
\Pi^{\mathrm{vol}} \equiv \Pi^{* \mathrm{vol}}\left(D_{33}, \bar{D}\right)=\frac{2 \bar{\sigma}}{\sqrt{3} L^{2}} \int_{R}^{L} \sqrt{\left(D_{33} \frac{L^{2}}{r^{2}}\right)^{2}+\bar{D}^{2}} r \mathrm{~d} r
$$


which can be evaluated conveniently using the change of variable $u \equiv L^{2} / r^{2}$ :

$$
\Pi^{\mathrm{vol}}=\frac{\bar{\sigma}|\bar{D}|}{\sqrt{3}} \int_{1}^{1 / \chi^{2}} \sqrt{1+\zeta^{2} u^{2}} \frac{\mathrm{d} u}{u^{2}} \quad, \quad \zeta \equiv \frac{D_{33}}{|\bar{D}|}
$$

(Note that $|\zeta| \leq 1 / \sqrt{3}$ ). The above integral emerges in various related problems, beginning with the Gurson model as revisited by Benzerga and Leblond (2010) and its extensions, for example Benzerga and Besson $(2001)^{1}$. Thus, combining the volume term in (16) with the surface term in (10) one finally obtains:

$$
\Pi=\frac{\bar{\sigma}|\bar{D}|}{\sqrt{3}}\left[\zeta \sinh ^{-1}(\zeta u)-\sqrt{\frac{1}{u^{2}}+\zeta^{2}}\right]_{1}^{1 / \chi^{2}}+\left|D_{33}\right| \Sigma^{\text {surf }}
$$

with $\Sigma^{\text {surf }}$ given by $(10)_{2}$. The dissipation function in (17) is not differentiable, just like the corresponding estimate from Torki et al. (2015), see their equation (32). However, unlike the estimate in (Torki et al., 2015), the above equation provides a strict upper bound to the plastic dissipation.

\subsection{UPPER-BOUND CRITERION}

Using the fundamental inequality of limit analysis (3) it can be shown that the upper-bound effective yield surface associated with (17) contains singular parts and regular ones with no vertices. The general procedure for determining the various regions in stress space follows that of Torki et al. (2015).

First, since the dissipation function $\Pi$ depends only on $D_{33}$ and $D_{31}$ the effective yield condition according to (3) or (5) will not depend on $\Sigma_{11}, \Sigma_{22}$ and $\Sigma_{12}$. Indeed by the normality flow rule $D_{i j}=\dot{\Lambda} \partial \Phi / \partial \Sigma_{i j}$ where $\Phi$ is the sought effective yield function and $\dot{\Lambda} \geq 0$ the plastic multiplier; thus, the coalescence conditions $D_{11}=D_{22}=D_{12}=0$ entail independence of the yield condition vis-a-vis the above-mentioned stress components.

Next, to obtain the singular parts one ought to resort to the primitive definition of the reversibility domain $\mathscr{C}$ in (3). The reasoning for doing so is intricate (see Appendix A) and is based on a graphical method. The result, however, is quite simple. Indeed, the yield locus is defined by:

$$
\left|\Sigma_{31}\right|=\left(1-\chi^{2}\right) \bar{\tau} ; \quad\left|\Sigma_{33}\right| \leq \Sigma^{\text {surf }}
$$

where $\bar{\tau}=\bar{\sigma} / \sqrt{3}$ is the shear yield strength of the matrix. This equation means that for the indicated range of normal stresses $\Sigma_{33}$, the effective yield function is independent of the normal stress and the shear yield stress is obtained by a simple rule of mixture between the yield stresses in the matrix and the void, since $\chi^{2}$ is exactly the porosity in the band. In what follows, we shall denote $\mathcal{T}=\left(1-\chi^{2}\right) \bar{\tau}$.

On the other hand, the regular parts require a totally different treatment, which is streamlined in Appendix B. The technical part involves eliminating parameter $\zeta$ defined by $(16)_{2}$ to obtain an explicit expression of the effective yield function, which is given by equation (B-13).

The equations of the upper-bound model are recapitulated herein for ease of reference:

$\Phi(\Sigma ; \chi, W)= \begin{cases}\frac{\mathcal{B}^{2}}{\bar{\tau}^{2}}+2 f_{\mathrm{b}} \cosh \left(\frac{\left|\Sigma_{33}\right|-\Sigma^{\text {surf }}}{\bar{\tau}}-\sqrt{\left.3 \frac{\mathcal{B}^{2}-\Sigma_{31}^{2}}{\bar{\tau}^{2}}\right)}-\left(1+f_{\mathrm{b}}^{2}\right)\right. & \text { for }\left|\Sigma_{33}\right| \geq \Sigma^{\text {surf }} \\ \frac{\Sigma_{31}^{2}}{\mathcal{T}^{2}}-1 & \text { for }\left|\Sigma_{33}\right| \leq \Sigma^{\text {surf }}\end{cases}$

\footnotetext{
${ }^{1}$ In equation (49) of Benzerga and Besson (2001) the term $\sigma_{1} / b^{2}$ appears as a typo and should be replaced with $h \sigma_{1} D_{m}$. Also, the bound of the integral should read $\xi / f$. The same typos slipped in equation (6.11) of Benzerga and Leblond (2010).

${ }^{2}$ Having assumed normality at the microscopic scale, equation (2), macroscopic normality is a rigorous consequence of the combination of effective properties and limit-analysis (Benzerga and Leblond, 2010).
} 
where $\bar{\tau}=\bar{\sigma} / \sqrt{3}$ is the shear yield strength, $f_{\mathrm{b}}=\chi^{2}$ is the porosity within the plastically-deformable band, and

$$
\begin{aligned}
& \Sigma^{\text {surf }}(\chi, W)=\frac{\chi^{3}-3 \chi+2}{3 \chi W} \bar{\tau} \\
& \mathcal{T}=\left(1-\chi^{2}\right) \bar{\tau} \\
& \frac{\mathcal{B}^{2}}{\bar{\tau}^{2}}=\frac{5}{3}+\chi^{4}-\frac{2}{3} \sqrt{4+12 \chi^{4}-3 \frac{\Sigma_{31}^{2}}{\bar{\tau}^{2}}}
\end{aligned}
$$

In general, the shear stress can be resolved into two components so that $\Sigma_{31}^{2}$ should be replaced with $\Sigma_{31}^{2}+$ $\Sigma_{32}^{2}$ everywhere in the above expressions.

By way of comparison, the equations of the approximate (not bound-preserving) criterion derived by Torki et al. (2015) are:

$$
\Phi(\Sigma ; \chi, W)= \begin{cases}\left(\frac{\left|\Sigma_{33}\right|-\Sigma^{\text {surf }}}{\Sigma^{\text {vol }}}\right)^{2}+\frac{\Sigma_{31}^{2}+\Sigma_{32}^{2}}{\mathcal{T}^{2}}-1 & \text { for }\left|\Sigma_{33}\right| \geq \Sigma^{\text {surf }} \\ \frac{\Sigma_{31}^{2}+\Sigma_{32}^{2}}{\mathcal{T}^{2}}-1 & \text { for }\left|\Sigma_{33}\right| \leq \Sigma^{\text {surf }}\end{cases}
$$

where

$$
\Sigma^{\mathrm{vol}}(\chi)=\left(2-\sqrt{1+3 \chi^{4}}+\ln \frac{1+\sqrt{1+3 \chi^{4}}}{3 \chi^{2}}\right) \bar{\tau}
$$

In both models (upper-bound and approximate) the yield surface is smooth, i.e., the transition between the two parts is vertex-free; see Appendix A. Also, note that the effective yield stress in shear is much smaller than that predicted by a Gurson-like model given that $\chi^{2} \equiv f_{\mathrm{b}}>f$.

\section{Cell-Model Calculations}

Previously, Torki et al. (2015) presented comparisons between their approximate model and the micromechanical calculations of Tekog̃lu et al. (2012), which were carried out for tetragonal cells containing spheroidal voids. In order to assess the predictive capabilities of the upper-bound model, calculations have now been carried out for the very same unit cell used in the development of the model, Fig. 1. Thereupon, a special small-strain finite element framework is employed which is intended to be the numerical equivalent of the theory of limit analysis; see also Madou and Leblond (2013). A classical consequence of limit-analysis is that elastic strain rates vanish when the limit load is reached. Therefore, the elastic moduli disappear from the equations and may be chosen arbitrarily, and in turn plasticity imposes an incompressible velocity field on the material. In this study, in order to mimic such a velocity field, a high value of Poisson's ratio is enforced $(\nu=0.49)$. Thus the matrix material is modeled as nearly isochoric-elastic ideal-plastic. Also, the yield strength to Young's modulus ratio is taken to be $\bar{\sigma} / E=0.0002$. All calculations were carried out using ABAQUS (Version 6.12) with the option of geometric nonlinearity switched off.

Inasmuch as the calculations are meant to validate the analytical model, it is emphasized that the same cylindrical geometry of Fig. 1 is used to avoid any ambiguity in the comparisons. One difficulty in making this choice is that strictly periodic boundary-conditions cannot be imposed on the cylindrical cell, since it does not truly represent a unit cell in a periodic medium but only "mimics" such a cell. Instead, "quasiperiodic" boundary conditions are prescribed drawing inspiration from rigorous periodicity. Consider one half of the cell (Fig. 2) with symmetry conditions imposed on the meridian plane. Anywhere in a periodic 


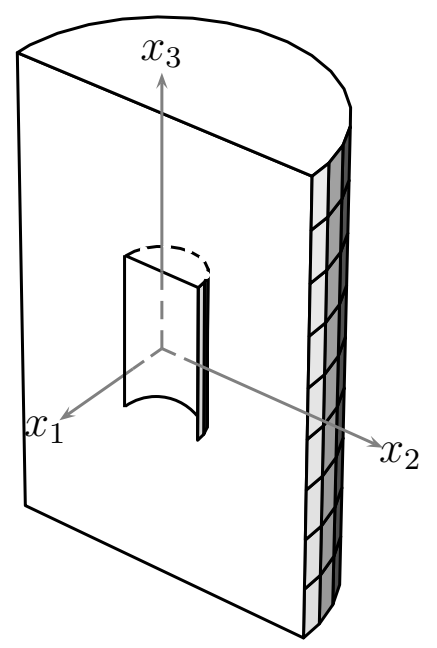

Figure 2: One half of a cylindrical cell.

cell, the displacement $\mathbf{u}$ at field point $\mathbf{x}$ would write:

$$
\mathbf{u}(\mathbf{x})=(\mathbf{E}+\mathbf{\Omega}) \cdot \mathbf{x}+\tilde{\mathbf{u}}(\mathbf{x})
$$

where $\mathbf{E}$ is the macroscopic strain tensor, $\boldsymbol{\Omega}$ the (skew-symmetric) macroscopic rotation tensor and $\tilde{\mathbf{u}}$ a periodic field. For any two points in periodic correspondence one would therefore have:

$$
\Delta \mathbf{u}=(\mathbf{E}+\mathbf{\Omega}) \cdot \Delta \mathbf{x}
$$

where $\Delta \mathbf{u}$ is the difference in displacement between the points separated by the vector $\Delta \mathrm{x}$. For a cylindrical cell equation (24) cannot be applied to pairs of points in periodic correspondence since such pairs do not exist. We shall impose instead conditions similar to (24) to specific, carefully selected pairs of surface points. It is in that sense that such conditions are termed "quasi-periodic".

Specifically, the macroscopic strain enforced on the cell is represented by the tensor:

$$
\mathbf{E}=E_{11}\left(\mathbf{e}_{1} \otimes \mathbf{e}_{1}+\mathbf{e}_{2} \otimes \mathbf{e}_{2}\right)+E_{33} \mathbf{e}_{3} \otimes \mathbf{e}_{3}+E_{31}\left(\mathbf{e}_{1} \otimes \mathbf{e}_{3}+\mathbf{e}_{3} \otimes \mathbf{e}_{1}\right)
$$

or in matrix form

$$
\mathbf{E}=\left[\begin{array}{ccc}
E_{11} & 0 & E_{31} \\
0 & E_{11} & 0 \\
E_{31} & 0 & E_{33}
\end{array}\right]
$$

where

$$
\begin{aligned}
& E_{11}=E_{22} \equiv \ln \left(\frac{L}{L_{0}}\right) \approx \frac{U_{1}}{L_{0}} \\
& E_{33} \equiv \ln \left(\frac{H}{H_{0}}\right) \approx \frac{U_{3}}{H_{0}} \\
& E_{31} \equiv \frac{U_{\mathrm{t}}}{2 H_{0}}
\end{aligned}
$$

Here, $U_{1}$ denotes a prescribed displacement on the lateral surface (see Appendix C for details), whilst $U_{3}$ and $U_{\mathrm{t}}$ are, respectively, the normal and tangential displacements prescribed on the top surface. 
On the other hand, the macroscopic rotation tensor must be of the form:

$$
\boldsymbol{\Omega}=\Omega_{31}\left(\mathbf{e}_{3} \otimes \mathbf{e}_{1}-\mathbf{e}_{1} \otimes \mathbf{e}_{3}\right)
$$

The simplest choice for $\Omega$ in (24) would be to take $\Omega=\mathbf{0}$. However, this would entail a vertical displacement on the lateral surface. To avoid this, one can choose $\Omega_{13}=-\Omega_{31}=E_{31}$. For the cylindrical cell considered, this choice will considerably simplify the formulation of multi-point constraint conditions.

In matrix form, the strictly periodic boundary conditions (24) now read:

$$
\left\{\begin{array}{l}
\Delta u_{1} \\
\Delta u_{2} \\
\Delta u_{3}
\end{array}\right\}=\left[\begin{array}{ccc}
E_{11} & 0 & 2 E_{31} \\
0 & E_{11} & 0 \\
0 & 0 & E_{33}
\end{array}\right]\left\{\begin{array}{l}
\Delta x_{1} \\
\Delta x_{2} \\
\Delta x_{3}
\end{array}\right\}
$$

They are replaced by the following quasi-periodic conditions:

- On the top surface, $\Delta \mathbf{u}=\mathbf{u}\left(x_{1}, x_{2}, H\right)-\mathbf{u}\left(x_{1}, x_{2},-H\right)$ and $\Delta \mathbf{x}^{T}=\{0,0,2 H\}$ so that:

$$
\left\{\begin{array}{l}
\Delta u_{1}=4 E_{31} H \\
\Delta u_{2}=0 \\
\Delta u_{3}=2 E_{33} H
\end{array}\right.
$$

- On the plane $O x_{2} x_{3}$,

$$
u_{1}\left(0, x_{2}, x_{3}\right)=0
$$

- On the lateral surface $\left(x_{1}^{2}+x_{2}^{2}=L^{2},-H \leq x_{3} \leq H\right)$ multi-point constraints are imposed so that the nodes lying on a semi-circle remain on a semi-circle of radius consistent with the prescribed value of $E_{11}$. Let $\mathbf{u}^{\text {ref }}$ be the displacement of some reference node on the semi-circle at some height $x_{3}$, say $\mathbf{x}^{T}=\left\{L, 0, x_{3}\right\}$ and $\Delta \mathbf{u}=\mathbf{u}\left(x_{1}, x_{2}, x_{3}\right)-\mathbf{u}^{\text {ref }}$, then:

$$
\left\{\begin{array}{l}
\Delta u_{1}=E_{11}\left(x_{1}-L\right) \\
\Delta u_{2}=E_{11} x_{2} \\
\Delta u_{3}=0
\end{array}\right.
$$

To simulate coalescence states whereby rigid zones preclude lateral straining we take $E_{11}=0$, hence $U_{1}=0$. Under such circumstances, conditions (31) state that the circles move rigidly.

In theory, the quasi-periodic boundary conditions are most simply defined by (29)-(31). In practice, however, it is of interest to employ only a quarter of the cell to further reduce the computation time. The corresponding boundary conditions have been worked out by Tekog̃lu (2014) for an tetragonal cell and have been adapted to the cylindrical cell as detailed in Appendix C. In a given calculation the displacements $U_{3}$ and $U_{\mathrm{t}}$ are imposed and assigned values to cause plastic strains that are large enough compared with elastic strains (see Tekog̃lu et al. (2012), Madou and Leblond (2013) for further details). The ratio between the shear and normal stresses is governed by the $U_{\mathrm{t}} / U_{3}$ ratio.

For each choice of the pair $(\chi, W)$ the critical stress values are, in principle, determined in a single-step calculation. The time step needs to be large enough to ensure that the limit load is reached. In practice, this is achieved within the first few increments (5 to 10) of the loading step. The absolute values of $U_{3}$ and $U_{\mathrm{t}}$ have no effect on the critical stress values.

The critical conditions on the stresses for attainment of the limit load are insensitive to the height $H$ of the cell, hence to $\lambda$. For this reason, $H$ is adjusted so as to reduce computational cost. On the other hand, the 
height $H$ must be large enough to guarantee the possible presence of rigid zones above and below the void ${ }^{3}$. In most cases, the void fully fits into the unit cell when the cell aspect ratio $\lambda$ is taken as unity. Yet, for some $(\chi, W)$ pairs, the void would protrude, and thus $\lambda$ ought to take other values. Different $\lambda$ ratios were thus adopted for varying $(\chi, W)$ pairs, as shown in Table 1 . For each $(\chi, W)$ pair, 18 different displacement ratios were imposed, obeying the relation $U_{\mathrm{t}} / U_{3}=k / 2$, where $k=0,2,3, \ldots, 10,20,30,40,80,120,160,200$. A larger $U_{\mathrm{t}} / U_{3}$ ratio induces a smaller ratio $\Sigma_{33}^{\text {coal }} / \Sigma_{31}^{\text {coal }}$ of the stresses at coalescence and vice versa (note that $\Sigma_{31}=0$ for $\left.U_{\mathrm{t}} / U_{3}=0\right)$.

\begin{tabular}{ccc}
$W$ & $\chi$ & $\lambda$ \\
\hline 0.1 & 0.4 & 0.5 \\
0.1 & 0.6 & 0.5 \\
1.5 & 0.4 & 1.2 \\
1.5 & 0.6 & 1.5 \\
2.0 & 0.4 & 1.6 \\
2.0 & 0.6 & 2.0 \\
2.5 & 0.4 & 2.0 \\
2.5 & 0.6 & 2.5 \\
3.0 & 0.4 & 2.4 \\
3.0 & 0.6 & 3.0 \\
\hline
\end{tabular}

Table 1: $W-\chi$ values used in the cell model calculations having $\lambda \neq 1$.

Figure 3 shows two typical meshes, used for a void aspect ratio $W=0.5$ and two values of the ligament parameter $\chi=(0.4,0.6)$. Each mesh consists of 20-node quadratic brick elements with reduced integration

(a)

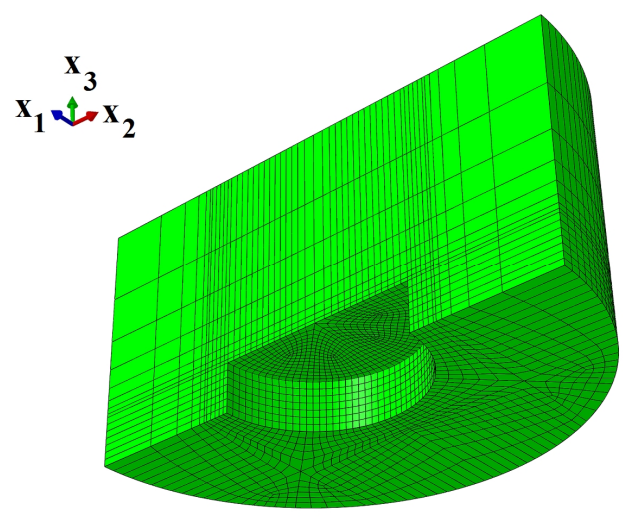

(b)

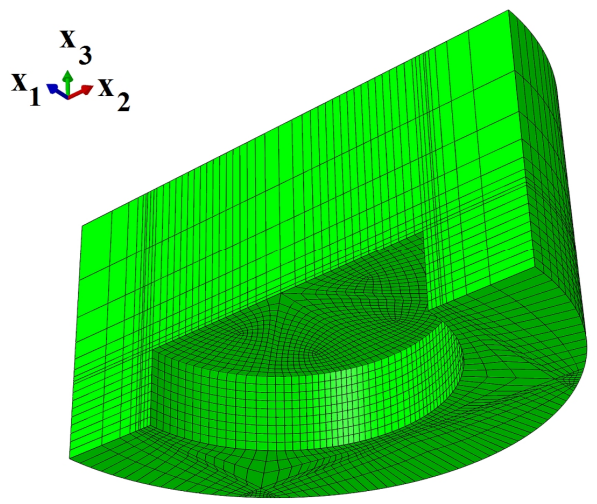

Figure 3: Typical meshes used for $W=0.5$ and: (a) $\chi=0.4$, (b) $\chi=0.6$.

(C3D20R in the ABAQUS element library). A coarser mesh is utilized outside the ligament where the behavior is quasi-rigid. Some calculations have been performed with both C3D8, as in (Tekog̃lu et al., 2012), and C3D20R elements types. Differences were small, but C3D20R elements were found to provide more accurate results with fewer elements.

\footnotetext{
${ }^{3}$ Also noteworthy is that the rigid zones above and below the void ought to be large enough to set grounds for localization to occur in the $x_{1}-x_{2}$ plane, referred to as "internal necking", as assumed in the present work. If the void is very close to the top (and bottom) surfaces of the unit cell, coalescence occurs in the $x_{2}-x_{3}$ plane (primarily named as a "necklace-type" coalescence by Benzerga (2000)), which is out of the scope of this investigation.
} 
Average stresses over the cell are defined as usual, $\Sigma_{i j}=(1 / \Omega) \int_{\Omega-\omega} \sigma_{i j} \mathrm{~d} V$ with $\Omega$ and $\omega$ denoting the volumes of the cell and the void, respectively, as before. These average stresses are computed using the discretized formula:

$$
\Sigma_{i j}=\sum_{n=1}^{N} \sum_{m=1}^{M}\left(\sigma_{i j}\right)_{n}^{m} v_{n}^{m}
$$

where $N$ is the total number of elements, $M$ the number of Gauss points per element (here $M=8$ ), and $v_{n}^{m}=V_{n}^{m} / \Omega$ the volume fraction assigned to integration point $m$ within element $n$. The components of interest are $\Sigma_{33}$ and $\Sigma_{31}$, all others either are zero or do not affect the limit load in the coalescence regime.

\section{Results and Discussion}

\subsection{UPPER-BOUND VERSUS APPROXIMATE YIELD LOCI}

Representative yield loci corresponding to the upper-bound criterion (19) are shown in Fig. 4 as solid lines for several values of the $(\chi, W)$ pair. The porosity in the band takes on values between $f_{\mathrm{b}}=0.0625$ and

(a)

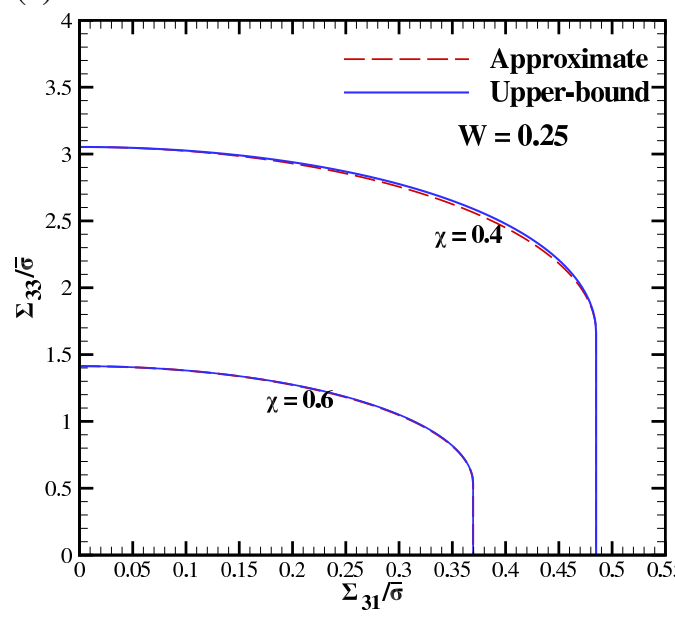

(c)

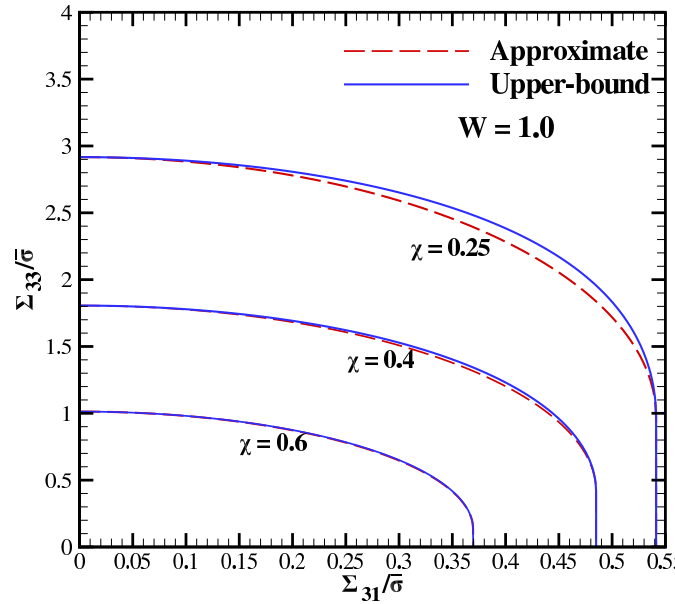

(b)

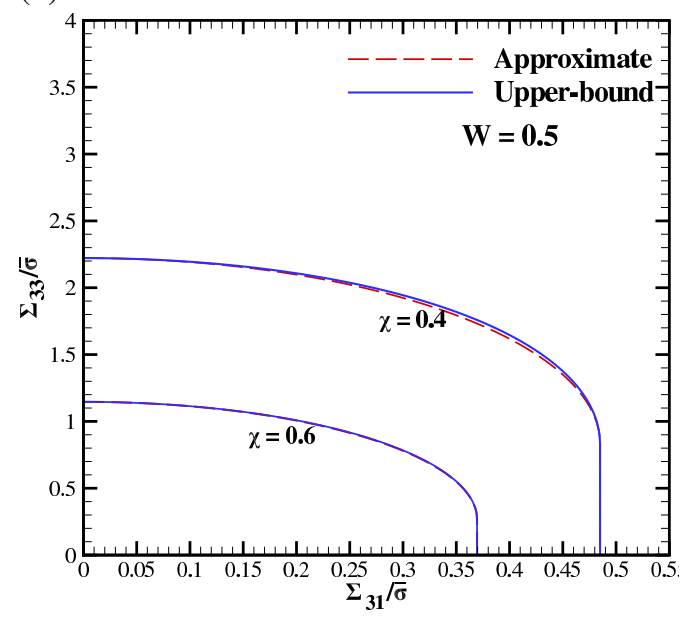

(d)

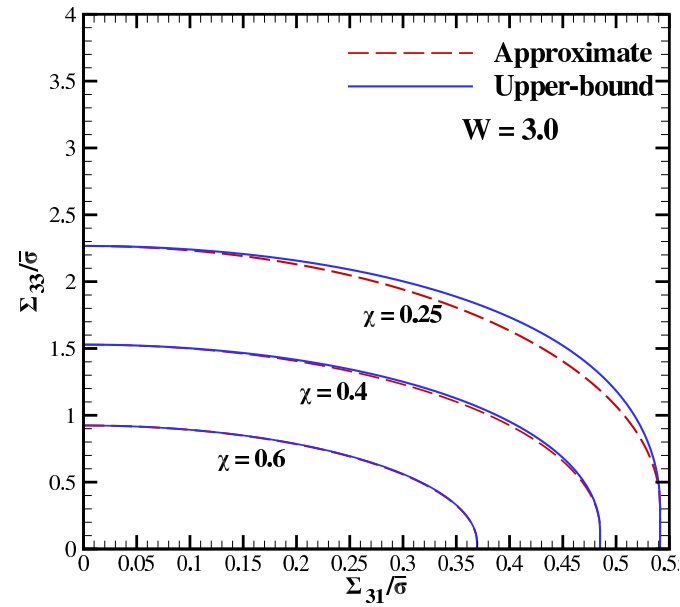

Figure 4: Effective yield loci in the $\Sigma_{33}-\Sigma_{31}$ plane - comparison between the upper-bound estimate and its approximate counterpart (as derived in Torki et al. (2015)) for several microstructural parameters $(\chi, W)$. 
0.36 for the extreme cases shown of $\chi=0.25$ and 0.6 , respectively. Corresponding values of the total porosity fall between $f \approx 0.004$ and $\approx 0.2$ depending on the void aspect ratio and taking $\lambda=1$ where appropriate. Previous work in the literature shows that for $\chi<0.2$, yield loci given by (19) are unlikely to be physical, because strain concentration within the intervoid ligaments does not occur (Benzerga, 2002). In such cases, Gurson-like potentials are more likely to prevail. The case $\chi=0.25$ is not shown in Figs.4a,b because for flat voids and $\chi<0.3$ coalescence is unlikely (see Table 1 in (Benzerga, 2002)). In all, the vertical straight parts represent the singular portions of the yield loci. Such parts are not physical, as they follow from considering discontinuous trial velocity fields. They occupy an increasingly small portion as $\chi$ increases so that the criterion resembles more and more an elliptic one in the space of normal and shear stresses.

For comparison, the approximate yield loci of Torki et al. (2015) defined by (21) are also shown dashed in Fig. 4. The singular parts are common to both models. The predictions differ only on the regular curved parts. While the upper-bound preserving curve is invariably exterior to the elliptic approximation, the two evaluations are always close to each other, especially for larger values of $\chi$. The key observation, therefore, is that the elliptic approximation is quite good over a wide range of internal parameters.

The fact that both models lead to very close predictions can be rationalized as follows. As mentioned above, the two models share the same singular parts; in particular the end points (for $\Sigma_{33}=\Sigma^{\text {surf }}$ ) are the same in the two models; also see Appendix B. Furthermore, the two criteria meet on the $\Sigma_{33}$ axis, that is in the absence of shear. (Although not obvious, this property is demonstrated in Appendix A; its basic explanation lies in the fact that approximation (13) becomes exact for $D_{31}=0$ ). In short, because both loci must meet at the intersections as well as on the $\Sigma_{33}$ axis, and because they are both convex, they must lie quite close to each other.

\subsection{COMPARISON With NumericAl RESUlts}

Figure 5 depicts contours of equivalent plastic strain at the onset of localization obtained in the finite element simulations, for two $(\chi, W)$ pairs and various values of shear- to normal-strain ratios. The figure illustrates that plastic deformation is diffuse in the plastically-deformable ligament, and it spreads over part of the ligament $(\mathrm{a}-\mathrm{d})$ or its entirety $(\mathrm{e}, \mathrm{f})$. In the mathematical model, however, it has been presumed that the whole ligament yields, which leads to an overestimation of the limit load. Hence, the analytical model preserves the upper-bound character. Moreover, plastic strains are negligible in the region above the void for all tensionshear combinations. This supports the underlying assumption of rigidity within the parts located above and below the ligament in developing the model (see Fig. 1).

Figure 6 shows the comparison between the cell-model calculations (points) and the upper-bound yield criterion (19) for various combinations of $\chi$ and $W$. Other numerical results were obtained but they are not shown for brevity. It is thus verified that the yield locus predicted by the model is always exterior to that determined numerically. In addition, the predicted locus is reasonably close to the exact one considering the fact that the model does not involve any adjustable parameter. This is especially true for void aspect ratios about unity or larger. However, differences may be noted in the some cases. For instance, in the limit $W \rightarrow 0$ of a penny-shape crack, the predicted coalescence stress in the absence of shear wrongly diverges, although not shown in Fig. 6 The singular behavior of the model in this limit has been discussed previously. The corresponding coalescence mechanism is arguably not by internal necking. In this case, modeling the localization would require adopting a thickness of the localization band larger than that of the void, which is nil in this case (Hure and Barrioz, 2016). Also, for small shear to normal stress ratios, differences are larger for smaller values of $\chi$. For large shear to normal stress ratios, differences are larger for larger values of $W$, irrespective of $\chi$. It is also worth noting that the predictions could hardly be improved in the pure shear case with $(W=0.5, \chi=0.4)$ or the pure tension case with $(W=3, \chi=0.6)$ without using overly sophisticated velocity fields. 
(a)

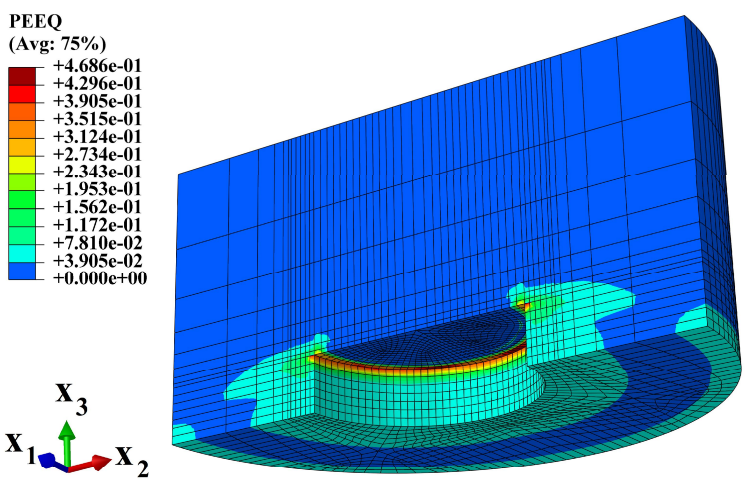

(c)

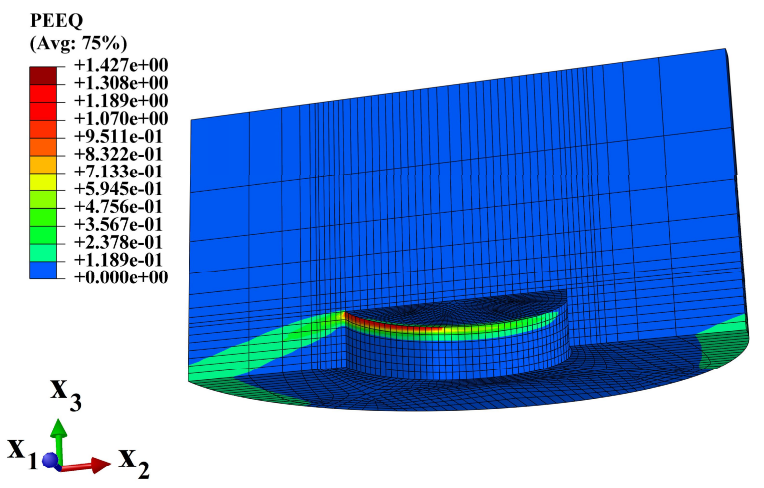

(e)

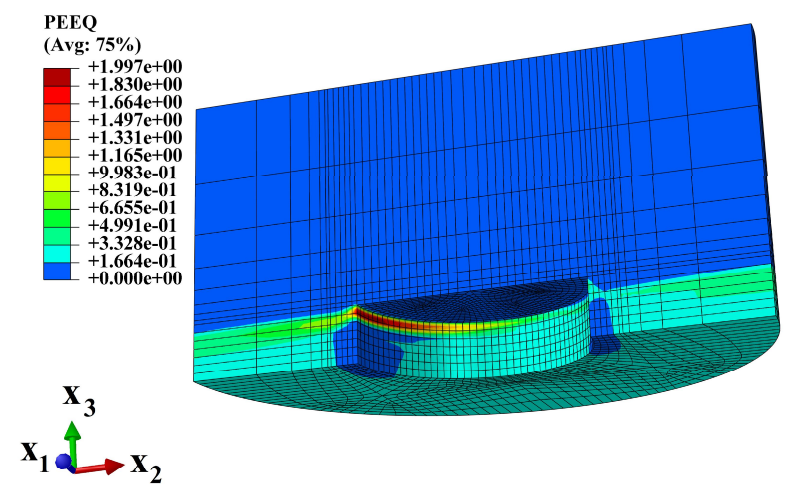

(b)

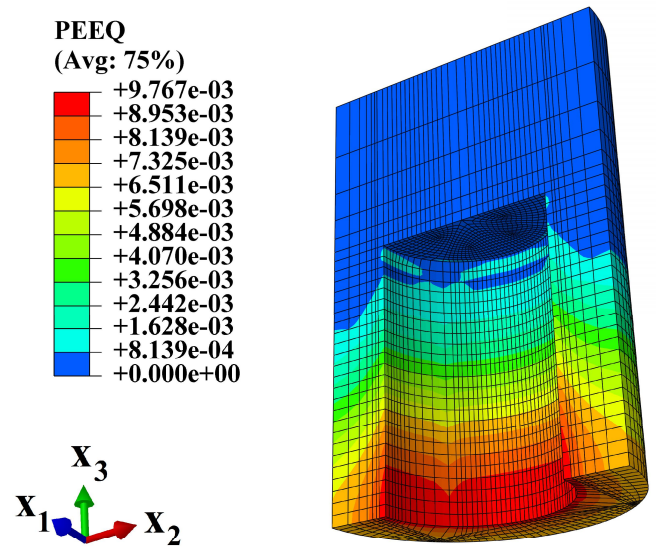

(d)
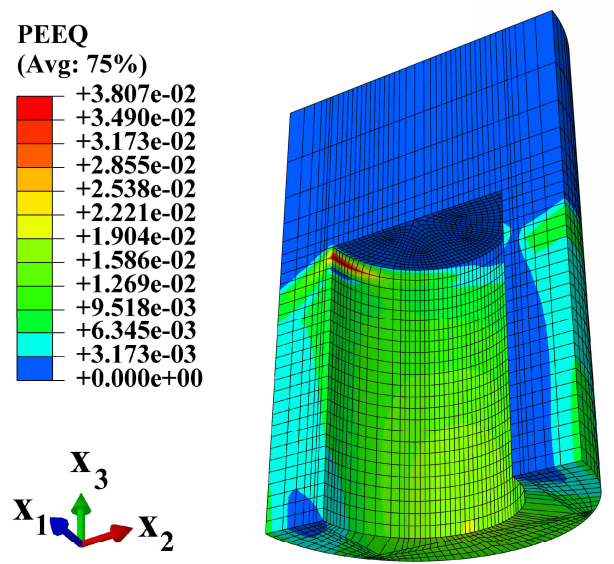

(f)
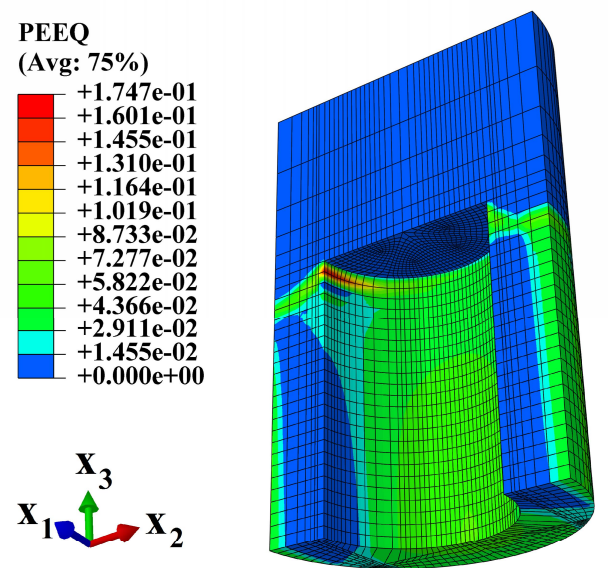

Figure 5: Examples of equivalent plastic strain distributions on initial configurations at the onset of internal necking localization, for: (a,b) $U_{t} / U_{3}=0$ (zero shear) and $(\chi, W)=\{(0.4,0.5),(0.6,3.0)\}$, (c,d) $U_{t} / U_{3}=5$ (intermediate shear) and $(\chi, W)=\{(0.4,0.5),(0.6,3.0)\}$, (e,f) $U_{t} / U_{3}=20$ (near-extremum shear) and $(\chi, W)=$ $\{(0.4,0.5),(0.6,3.0)\}$. 
(a)

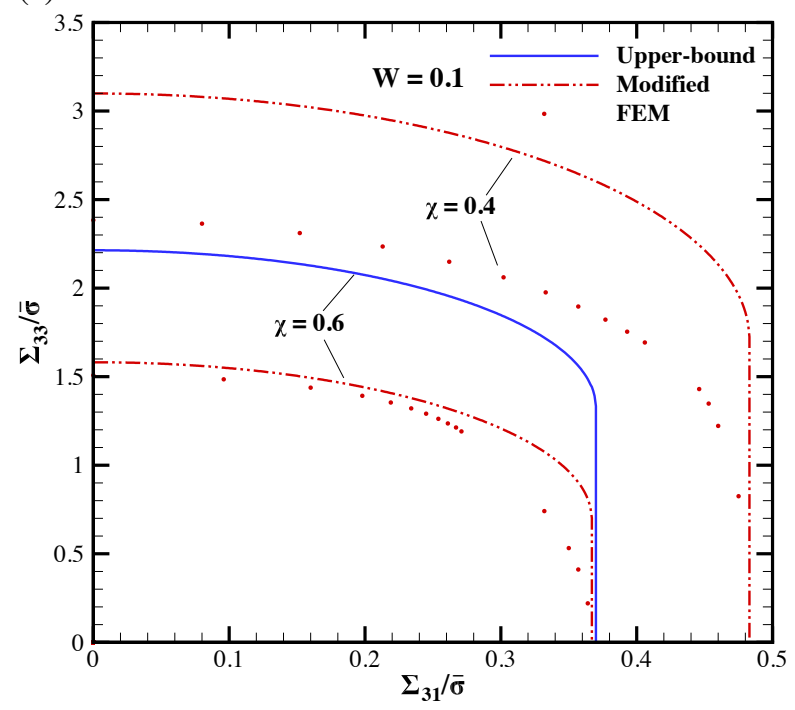

(c)

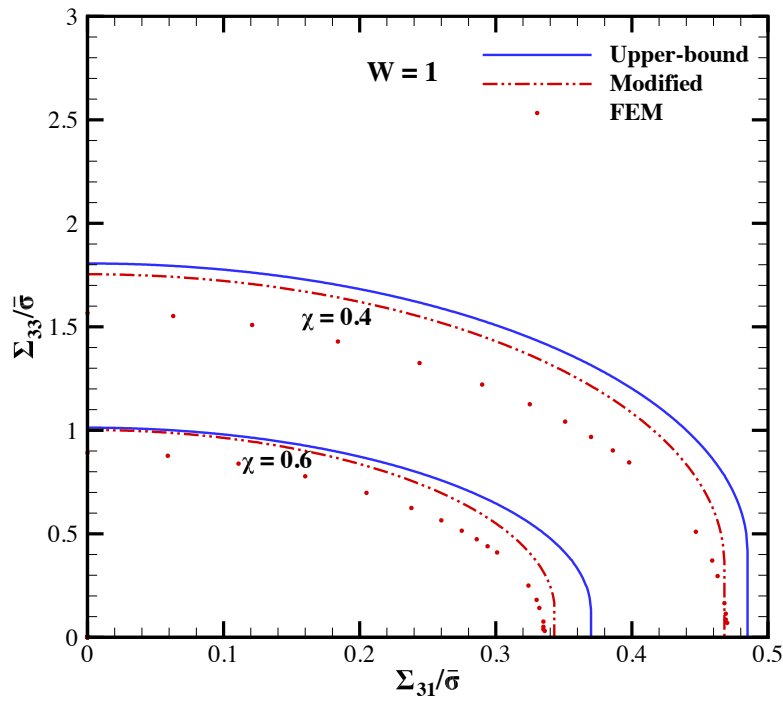

(b)

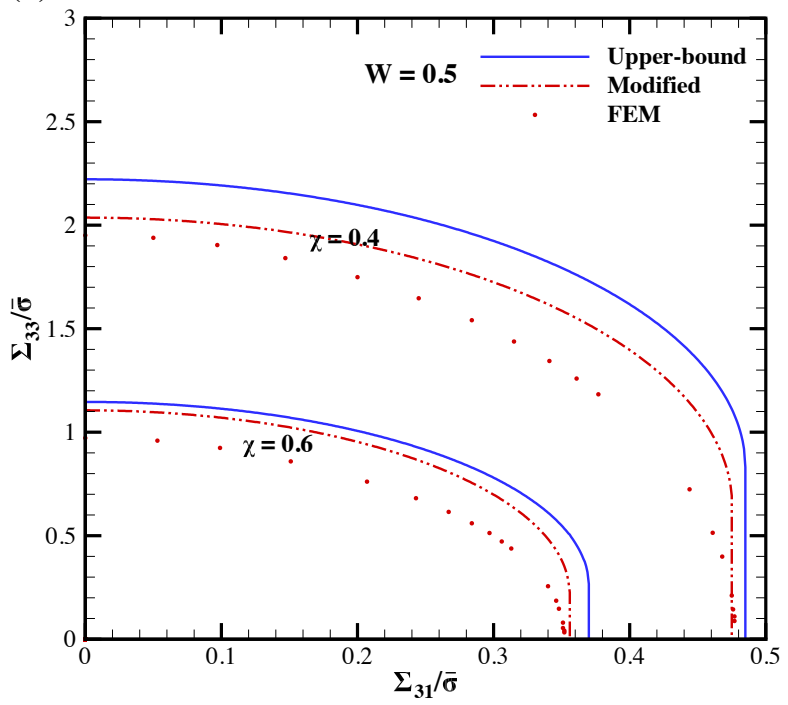

(d)

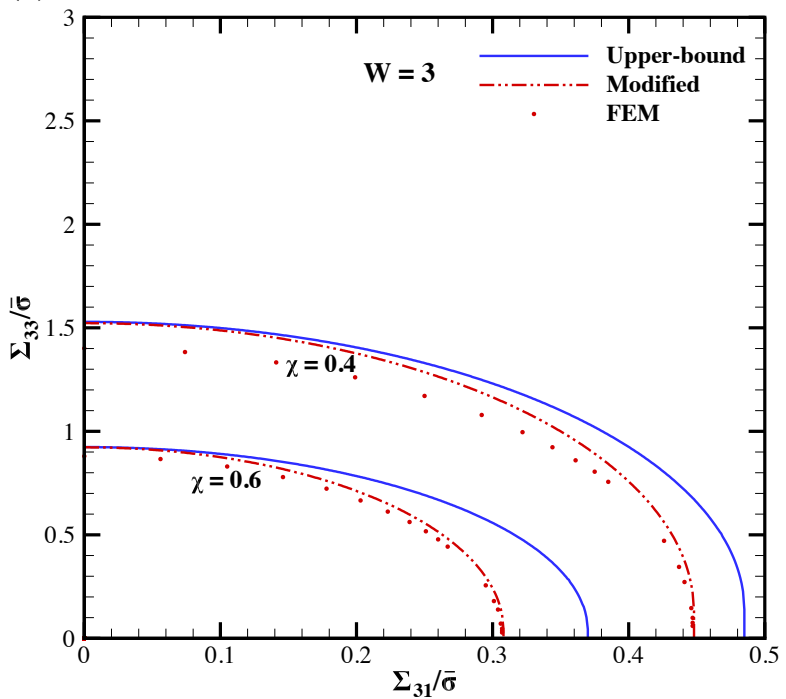

Figure 6: Comparison between the upper bound model (solid lines), modified model (dashed) and numerical yield loci (points) for void coalescence under combined tension and shear, for various values of microstructural parameters $W$ and $\chi$. The modification (described in Section 4.3) is made to improve the results for very oblate $(W \longrightarrow 0)$ and very prolate $(W \longrightarrow \infty)$ cavities. 
We emphasize that the presence of flat parts in the yield loci near the horizontal axis is a direct consequence of considering discontinuous trial velocity fields in the limit-analysis. Hence, they are not physical. This does not prevent the analytical and numerical criteria to be close to each other in this region. Note that in the absence of shear the improved model of Morin et al. (2015), which was based on continuous velocity fields, provides tighter upper bounds for several combinations of the internal parameters.

\subsection{Modified Model}

The discrepancies with respect to numerical results motivate a heuristic modification of the original model. The main discrepancies occur in two distinct cases, both of some practical importance: (i) very flat voids under conditions of dominant tension; (ii) very elongated voids under conditions of dominant shear. Some improvements have been proposed to remedy these aspects in the context of an approximate model (Torki et al., 2015). The same is attempted here for the upper-bound model. Such a heuristic modification unavoidably destroys the rigorous upper-bound character of the model. Again, however, the main implications of this modification are only for extremely flat voids in tension and elongated voids in shear.

Following the procedure explained in Appendix D, the derived criterion is modified as follows:

$\Phi(\boldsymbol{\Sigma} ; \chi, W)= \begin{cases}\frac{\mathcal{B}^{2}}{\bar{\tau}^{2}}+2 f_{\mathrm{b}} \cosh \left[\frac{\left|\Sigma_{33}\right|-t \Sigma^{\text {surf }}}{\bar{\tau}}-\sqrt{3 \frac{\mathcal{B}^{2}-\left(\Sigma_{31} / l\right)^{2}}{\bar{\tau}^{2}}}\right]-\left(1+f_{\mathrm{b}}^{2}\right) & \text { for }\left|\Sigma_{33}\right| \geq t \Sigma^{\text {surf }} \\ \left(\frac{\Sigma_{31}}{l \mathcal{T}}\right)^{2}-1 & \text { for }\left|\Sigma_{33}\right| \leq t \Sigma^{\text {surf }}\end{cases}$

where

$$
\frac{\mathcal{B}^{2}}{\bar{\tau}^{2}}=\frac{5}{3}+\chi^{4}-\frac{2}{3} \sqrt{4+12 \chi^{4}-3\left(\frac{\Sigma_{31}}{l \bar{\tau}}\right)^{2}}
$$

and $t$ and $l$ are parameters that can be adjusted on the basis of the cell model calculations of Section 3 . Formulae for these quantities, as functions of the internal parameters, are presented in Appendix D. The basic idea is to employ a homographic function of $W$ for $t$ so as to eliminate the singular behavior in the limit of penny-shaped cracks, and a corrective bilinear function for $l$ to improve the prediction for shear loading of elongated voids.

The example yield loci shown below are intended to compare the modified yield criterion with FEM results, as well as assess its putative upper-bound character. Figure 6 illustrates this comparison for various values of $\chi$ and $W$. The calibrated loci for Figs. 6c and 6d may be compared to Figs. 4c and 4d, respectively, to assess the effect of large values of $W$ on the maximum shear stress at coalescence. The largest difference between the modified model and the numerical results is obtained for very flat voids ( $W=0.1$ ) but only for $\chi=0.4$, Fig. 6 a. The proposed heuristic correction performs much better for larger values of $\chi$. More elaborate choices for the correction functions are possible, albeit at the expense of simplicity.

\section{Concluding Remarks}

An upper-bound model of dilatant plasticity has been developed based on limit analysis of a cylindrical elementary cell. The model consists of an effective yield criterion that is appropriate to the regime of void coalescence in materials failing by ductile damage accumulation. Incidentally, the model is also applicable to porous media with periodic distribution of pores, as in some cellular structures. In all applications, the model would represent certain portions of the yield locus and should be supplemented with a model that appropriately represents other portions where plasticity cannot be confined to intervoid ligaments. This 
results in a hybrid multi-surface approach (Benzerga et al., 2002). Alternatively, Morin et al. (2016) have recently developed a unified upper-bound model that describes both void growth and coalescence in the absence of shear. Their approach can be extended to account for shear effects.

In practice, the model can be used in two ways. If there is a finite set of localization planes, such as for periodic or clustered void distributions, then the coalescence criterion (19) may be checked, as is, on as many localization planes as dictated by the underlying void distribution. In this case, an anisotropic void growth model must be used prior to coalescence, e.g. (Keralavarma and Benzerga, 2010, Madou and Leblond, 2012). In such a hybrid multi-surface approach, some approximations would be necessary to account for the different elementary cells used prior to and after coalescence. To this end, the model should also be extended to include evolution equations of the state variables, notably to describe void rotation under shear-dominated loadings. Such work is underway and will be reported elsewhere. On the other hand, if the void distribution is considered as random then an isotropic version of the model may be developed by probing all possible angles in the orientation space (Leblond and Mottet, 2008). In this case, an isotropic void growth model, such as Gurson's, may be used prior to void coalescence.

The trial velocity fields used in the limit analysis are not as sophisticated as those recently considered by Morin et al. (2015) or Keralavarma and Chockalingam (2016). However, they present the considerable advantage of enabling an upper-bound result to be derived in closed form.

To further assess the model, cell-model calculations of a new type have been carried out using special boundary conditions, termed quasi-periodic as they simulate rigorous periodicity. The availability of such computational results made it possible to unequivocally validate the model as well as an earlier version that did not preserve the upper-bound character. The major conclusions are as follows:

- The present findings remedy for the uncertainties associated with the model recently derived by Torki et al. (2015) from two perspectives: (i) the mathematical approximation involved in the homogenization procedure is relaxed, so that the new model now preserves the rigorous upper-bound character; (ii) the numerical results used for validation of the model are carried out for a cell identical to that considered in its derivation. Although the new model is more complex than the previous one, it remains fully explicit (the yield criterion is still expressed in explicit, not parametric form).

- Salient features of the new model include the following: (i) The planar (singular and non-physical) parts apparent in the approximate effective yield locus of Torki et al. (2015) are identically retrieved in the new model. The curved (regular) parts, however, are exterior to their approximate counterparts; (ii) The transition between the two planar and curved zones is devoid of any corners; (iii) All parameters defining the yield surface are functions of the microstructural variables $\chi$ (ligament parameter) and $W$ (void aspect ratio).

- The availability of the upper-bound solution allows to check that the approximation previously introduced by Torki et al. (2015) did not introduce important errors.

- The discrepancies that were noted in the previous work between the approximate model and numerical results cannot be attributed to the uncontrolled approximation used, nor to the difference between the elementary cells used. Instead, the gap between analytical and numerical results can only follow from the choice of trial velocity fields. The quality of the estimate derived with the velocity fields chosen here may be improved using the kind of heuristic fit described in the paper.

- A heuristic modification to the model was proposed on the basis of the numerical results in order to better predict the onset of coalescence in practical applications. The modifications are two-fold. First, a correction is proposed in the limit of penny-shaped cracks, which reduces to the correction proposed by Torki et al. (2015) in the absence of shear loading. Second, a correction is introduced in the case of elongated voids under shear-dominated loading. 


\section{Acknowledgments}

MT and AAB acknowledge support from the National Science Foundation under Grant Number CMMI1405226. JBL acknowledges financial support from Institut Universitaire de France (IUF).

\section{Appendix A Singular parts of the yield surface}

The primitive definition of the reversibility domain $\mathscr{C}$ in (3) writes:

$$
\boldsymbol{\Sigma} \in \mathscr{C} \Leftrightarrow \forall \mathbf{D}, \quad \boldsymbol{\Sigma}: \mathbf{D} \leq \Pi
$$

Considering (17) and the non-zero components of $\mathbf{D}$, it may be recast as:

$$
\forall D_{33}, D_{31}, \quad \Sigma_{33} D_{33}+2 \Sigma_{31} D_{31} \leq \frac{\bar{\sigma}|\bar{D}|}{\sqrt{3}}\left[\zeta \sinh ^{-1}(\zeta u)-\sqrt{\frac{1}{u^{2}}+\zeta^{2}}\right]_{1}^{1 / \chi^{2}}+\Sigma^{\text {surf }}\left|D_{33}\right|
$$

with $\bar{D}$ defined by (14) and $\zeta=D_{33} /|\bar{D}|$. Focussing on non-negative values of $D_{31}$ only, (A-2) is rewritten as:

$$
\forall D_{33}, \forall D_{31} \geq 0, \quad-\Pi\left(D_{33}, D_{31}\right) \leq \Sigma_{33} D_{33}+2 \Sigma_{31} D_{31} \leq \Pi\left(D_{33}, D_{31}\right)
$$

where use has been made of (A-2) for the pair $\left(-D_{33},-D_{31}\right)$ and of the fact that $\Pi$ is an even function. Inequalities (A-3) are equivalent to some condition $\Phi\left(\Sigma_{33}, \Sigma_{31}\right) \leq 0$ where $\Phi$ is the sought yield function. Since $\Pi$ is positively homogeneous of degree 1 , (A-3) may be written in terms of the ratio $p=D_{33} / D_{31}$ as:

$$
\forall p \in \mathbb{R}, \quad-g(p) \leq f(p) \leq g(p)
$$

where the functions $f$ and $g$ are defined on $\mathbb{R}$ by:

$$
\begin{aligned}
& f(p)=\Sigma_{33} p+2 \Sigma_{31} \\
& g(p) \equiv \Pi(p, 1)=\bar{\sigma} \sqrt{\frac{3 p^{2}+4}{3}}\left[\frac{p}{\sqrt{3 p^{2}+4}} \sinh ^{-1}\left(\frac{p u}{\sqrt{3 p^{2}+4}}\right)-\sqrt{\frac{1}{u^{2}}+\frac{p^{2}}{3 p^{2}+4}}\right]_{1}^{1 / \chi^{2}}+\Sigma^{\text {surf }}|p|
\end{aligned}
$$

In writing (A-5) use has been made of the relationship:

$$
p=\frac{2 \zeta}{\sqrt{1-3 \zeta^{2}}}
$$

The function $g$ is convex, admits a minimum $g(0)=2 \mathcal{T}$, its graph has an angular point at its minimum, and admits straight asymptotes of slope $\pm \bar{\sigma}\left[\frac{1}{\sqrt{3}} \sinh ^{-1}\left(\frac{u}{\sqrt{3}}\right)-\sqrt{\frac{1}{u^{2}}+\frac{1}{3}}\right]_{1}^{1 / \chi^{2}}+\operatorname{sgn}\left(D_{33}\right) \Sigma^{\text {surf }}$ for $p \rightarrow \pm \infty$; see Fig. A-1a.

The yield surface is the boundary of the reversibility domain, now defined by (A-4). As such, the yield locus is the envelope of the straight lines $q=f(p)$ lying between the graphs of the functions $q=-g(p)$ and $q=g(p)$ and meeting one of them at some point, Fig. A-1c. To construct this locus, we only consider loadings with $\Sigma_{31} \geq 0$ due to point symmetry about the origin, and implement a graphical solution as follows.

Two cases are analyzed separately depending on the magnitude of the normal stress. Consider first the case $\left|\Sigma_{33}\right| \leq g^{\prime}\left(0^{+}\right)=\Sigma^{\text {surf }}$. As illustrated in Fig. A-1a, the slope of the straight line $q=f(p)$ is smaller 
(a)

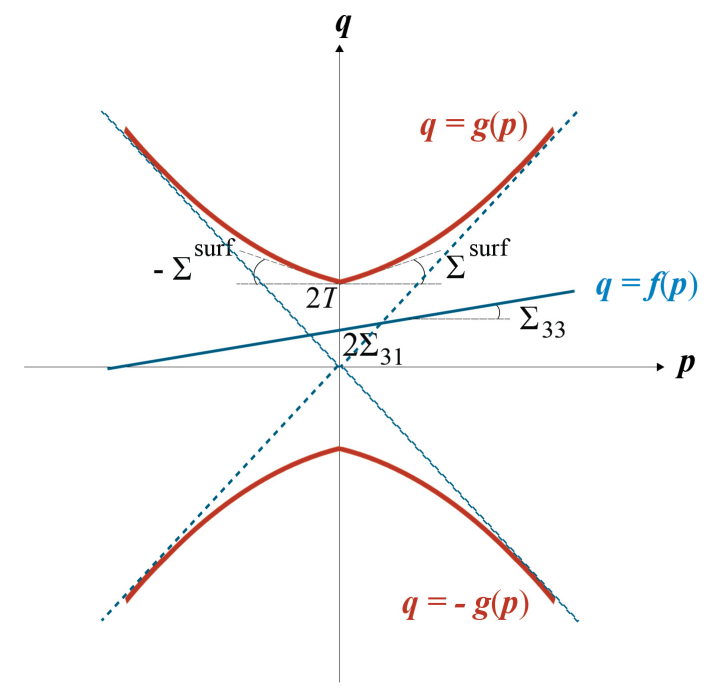

(c)

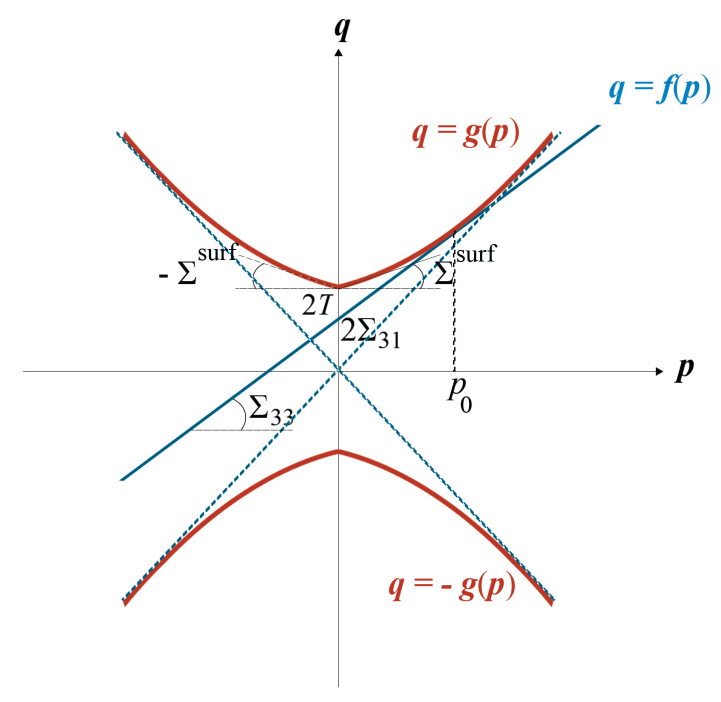

(b)

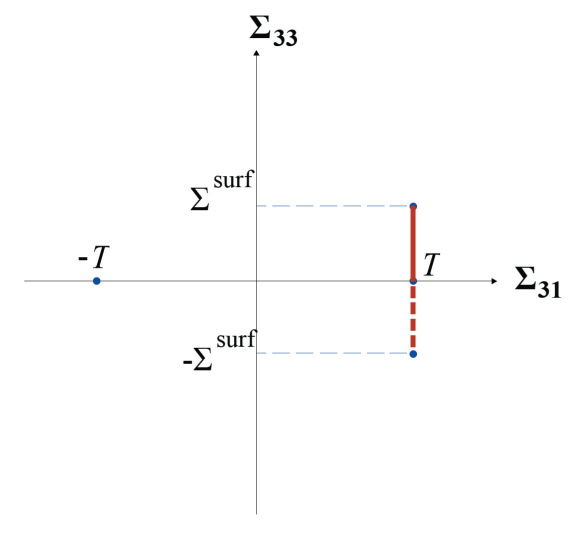

(d)

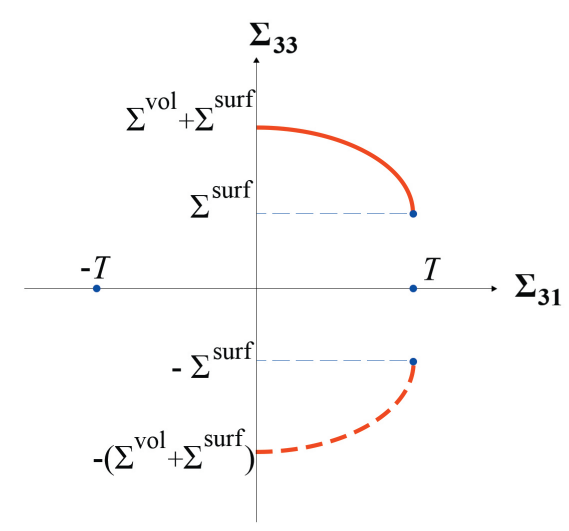

Figure A-1: Construction of the yield locus by a graphical method. (a) Illustration of inequalities (A-4) for $\left|\Sigma_{33}\right| \leq \Sigma^{\text {surf }}$; (b) corresponding (singular) part of yield locus. (c) Illustration for $\left|\Sigma_{33}\right| \geq \Sigma^{\text {surf }}$; (d) corresponding (regular) part of yield locus.

than that of the curve $q=g(p)$ at the origin so that the two curves do not cross each other provided that $f(0) \leq g(0)$, i.e. that $\Sigma_{31} \leq \mathcal{T}$. In this case, therefore, the yield condition is that $\Sigma_{31}=\mathcal{T}$, which is a constant (Fig. A-1b). The same condition holds for $g^{\prime}\left(0^{-}\right)<\Sigma_{33}<0$ by considering the intersections of lines $q=f(p)$ having negative slopes with the appropriate branches of the representative curves of the functions $g(p)$ and $-g(p)$.

Next, for $\left|\Sigma_{33}\right| \geq \Sigma^{\text {surf }}$ there must be a condition on the shear stress $\Sigma_{31}$ in terms of $\Sigma_{33}$ for the stress 
state to lie on the yield surface. Given $\Sigma_{33}$, the value of $\Sigma_{31}$ must be smaller than that which makes the line $q=f(p)$ tangent to the curve $q=g(p)$ (Fig. A-1c). Only in this case would the condition $f(p) \leq g(p)$ be ensured for every $p$. Finding the yield point $\left(\Sigma_{33}, \Sigma_{31}\right)$ then amounts to determining the value of $p$ for which there is tangency. The derivation is quite involved and was illustrated by Torki et al. (2015) in the case of the approximate dissipation $g(p)$. It is emphasized that in this case the dissipation function is differentiable and the procedure leads to a locus exactly given by (B-13). Fig. A-1d depicts the corresponding (regular) curved portion of the yield locus. For completeness, the portion corresponding to negative slopes of the line $q=f(p)$ is shown dashed in the figure.

Remark 1: In absence of shear Eq. (B-13), or equivalently Eq. $(19)_{1}$, predicts a limit load equal to $\Sigma^{\text {surf }}+\Sigma^{\mathrm{vol}}$, which is the same value as that obtained by Benzerga and Leblond (2014). Indeed, setting $\Sigma_{31}=0$ in $(19)_{1}$ one gets:

$$
\frac{\mathcal{B}^{2}}{\bar{\tau}^{2}}=\frac{5}{3}+f_{\mathrm{b}}^{2}-\frac{4}{3} \sqrt{1+3 f_{\mathrm{b}}^{2}}=\frac{1}{3}\left(\sqrt{1+3 f_{\mathrm{b}}^{2}}-2\right)^{2}
$$

which, after plugging in the yield function gives:

$$
\begin{gathered}
\cosh \left(\frac{\left|\Sigma_{33}\right|-\Sigma^{\text {surf }}}{\bar{\tau}}-\sqrt{3} \frac{\mathcal{B}}{\bar{\tau}}\right)=\mathcal{C} \quad, \quad \mathcal{C}=\frac{1+f_{\mathrm{b}}^{2}-(\mathcal{B} / \bar{\tau})^{2}}{2 f_{\mathrm{b}}}=\frac{2 \sqrt{1+3 f_{\mathrm{b}}^{2}}-1}{3 f_{\mathrm{b}}} \\
\text { hence } \mathcal{C}^{2}-1=\frac{1}{3}\left(\frac{2-\sqrt{1+3 f_{\mathrm{b}}^{2}}}{f_{\mathrm{b}}}\right) \\
\quad \frac{\left|\Sigma_{33}\right|-\Sigma^{\text {surf }}}{\bar{\tau}}-\sqrt{3} \frac{\mathcal{B}}{\bar{\tau}}=\cosh ^{-1} \mathcal{C}=\ln \left(\mathcal{C}+\sqrt{\mathcal{C}^{2}-1}\right)=\ln \frac{1+\sqrt{1+3 f_{\mathrm{b}}^{2}}}{3 f_{\mathrm{b}}} \\
\Longrightarrow \frac{\left|\Sigma_{33}\right|}{\bar{\tau}}=\frac{\Sigma^{\text {surf }}}{\bar{\tau}}+2-\sqrt{1+3 f_{\mathrm{b}}^{2}}+\ln \frac{1+\sqrt{1+3 f_{\mathrm{b}}^{2}}}{3 f_{\mathrm{b}}} \equiv \frac{\Sigma^{\text {surf }}}{\bar{\tau}}+\frac{\Sigma^{\text {vol }}}{\bar{\tau}}
\end{gathered}
$$

Remark 2: The upper-bound and approximate yield criteria given by Eqs (19) and (21), respectively, give the same yield condition in the absence of shear. In other words the loci meet on the $\Sigma_{33}$ axis. This property follows from the fact that approximation (13) is exact for $D_{31}=0$. Upon examination of the two criteria, Eqs (19) and (21), this seems strikingly surprising. Nevertheless, it was shown above that (19) does lead to $\Sigma_{33}=\Sigma^{\text {surf }}+\Sigma^{\text {vol }}$ when $\Sigma_{31}=0$ although $\Sigma^{\text {vol }}$ does not appear explicitly in (19). Obviously, this result is easier to establish by starting from the expression of the total dissipation. Indeed, if one sets $D_{31}=0$ or equivalently $\zeta=1 / \sqrt{3}$ in equation (17) then one gets:

$$
\begin{aligned}
\frac{\Sigma_{33}-\Sigma^{\text {surf }}}{\bar{\sigma}} & =\left[\frac{1}{\sqrt{3}} \sinh ^{-1}\left(\frac{u}{\sqrt{3}}\right)-\sqrt{\frac{1}{u^{2}}+\frac{1}{3}}\right]_{1}^{1 / \chi^{2}}=\frac{1}{\sqrt{3}}\left[2-\sqrt{1+3 \chi^{4}}+\ln \frac{1+\sqrt{1+3 \chi^{4}}}{3 \chi^{2}}\right] \\
& =\frac{\Sigma^{\mathrm{vol}}}{\bar{\sigma}}
\end{aligned}
$$

where use has been made of the identity: $\sinh ^{-1}(x)=\ln \left(x+\sqrt{x^{2}+1}\right)$ and that the left-hand side of the above equation is nothing but $\Pi^{* \mathrm{vol}} / D_{33}$ in the absence of shear.

Remark 3: The transition from the regular (curved) part to the singular (straight) part occurs without any vertex as shown by Torki et al. (2015). A geometric proof of this property was provided by Morin et al. (2016). So is the case because the first derivative of $\Phi$ with respect to $\Sigma_{31}$ from Eq. (19) $)_{1}$ would become 
unbounded at $\Sigma_{31}=\mathcal{T}$ or, equivalently, its derivative with respect to $\Sigma_{33}$ would vanish as follows. Letting $\left(\left|\Sigma_{33}\right|,\left|\Sigma_{31}\right|\right)=\left(\Sigma^{\text {surf }}, \mathcal{T}\right)$ would give:

$$
\begin{aligned}
& \frac{\mathcal{B}^{2}}{\bar{\tau}^{2}}=\frac{5}{3}+f_{\mathrm{b}}^{2}-\frac{2}{3} \sqrt{4+12 f_{\mathrm{b}}^{2}-3\left(1-f_{\mathrm{b}}\right)^{2}}=f_{\mathrm{b}}^{2}-2 f_{\mathrm{b}}+1=\left(1-f_{\mathrm{b}}\right)^{2} \\
& \text { hence } 3 \frac{\mathcal{B}^{2}-\Sigma_{31}^{2}}{\bar{\tau}^{2}}=\left(1-f_{\mathrm{b}}\right)^{2}-\left(1-f_{\mathrm{b}}\right)^{2}=0 \text { and } \frac{\left|\Sigma_{33}\right|-\Sigma^{\operatorname{surf}}}{\bar{\tau}}-\sqrt{\frac{\mathcal{B}^{2}-\Sigma_{31}^{2}}{\bar{\tau}^{2}}}=0 \\
& \Longrightarrow \frac{\partial \Phi}{\partial \Sigma_{33}}=\frac{\partial}{\partial \Sigma_{33}}\left(\frac{\mathcal{B}^{2}}{\bar{\tau}^{2}}\right)+2 f_{\mathrm{b}} \frac{\operatorname{sgn}\left(\Sigma_{33}\right)}{\bar{\tau}} \sinh \left(\frac{\left|\Sigma_{33}\right|-\Sigma^{\operatorname{surf}}}{\bar{\tau}}-\sqrt{\frac{\mathcal{B}^{2}-\Sigma_{31}^{2}}{\bar{\tau}^{2}}}\right)=0
\end{aligned}
$$

This demonstrates that the yield locus is devoid of any corners.

\section{Appendix B Regular parts of the yield surface}

On these, the dissipation function is differentiable so that equation (5) may be used. Since $\Pi$ is positively homogeneous of degree $1, \partial \Pi / \partial \mathbf{D}$ is positively homogeneous of degree 0 , i.e. $(\partial \Pi / \partial \mathbf{D})(\alpha \mathbf{D})=$ $(\partial \Pi / \partial \mathbf{D})(\mathbf{D})$, where $\alpha$ is an arbitrary positive real number. By way of consequence, $\partial \Pi / \partial \mathbf{D}$ depends only on the ratio $D_{31} / D_{33}$ of the two independent components of $\mathbf{D}$. This ratio can then, in principle, be eliminated between the two parametric equations of the yield locus; see Benzerga and Leblond (2010) for generalities.

In practice, however, there arises the difficulty that the obtained expression (17) involves a different ratio, $\zeta$, of $D_{33}$ to the composite strain-rate measure $\bar{D}$. Hence, proceed as follows. From (5) and using the variables $\left(D_{33}, \bar{D}\right)$ in lieu of $\left(D_{33}, D_{31}\right)$ obtain the nonzero stress components as:

$$
\begin{aligned}
& \Sigma_{33}=\frac{\partial \Pi}{\partial D_{33}}=\frac{\partial \Pi^{* \mathrm{vol}}}{\partial D_{33}}+\frac{\partial \Pi^{* \mathrm{vol}}}{\partial \bar{D}} \frac{\partial \bar{D}}{\partial D_{33}}+\operatorname{sgn}\left(D_{33}\right) \Sigma^{\text {surf }} \\
& \Sigma_{31}=\frac{1}{2} \frac{\partial \Pi}{\partial D_{31}}=\frac{1}{2} \frac{\partial \Pi^{* \mathrm{vol}}}{\partial \bar{D}} \frac{\partial \bar{D}}{\partial D_{31}}
\end{aligned}
$$

where the factor $1 / 2$ in the second equation is due to the fact that $D_{31}$ appears in fact twice in the actual dissipation function, as $D_{31}$ and $D_{13}$. In (B-1) appear stress-like auxiliary variables $\mathcal{A}$ and $\mathcal{B}$ :

$$
\begin{aligned}
& \mathcal{A} \equiv \frac{\partial \Pi^{* \mathrm{vol}}}{\partial D_{33}}=\frac{\bar{\sigma}}{\sqrt{3}}\left[\sinh ^{-1}(\zeta u)\right]_{1}^{1 / \chi^{2}} \\
& \mathcal{B} \equiv \frac{\partial \Pi^{* \mathrm{vol}}}{\partial \bar{D}}=-\operatorname{sgn}(\bar{D}) \frac{\bar{\sigma}}{\sqrt{3}}\left[\sqrt{\frac{1}{u^{2}}+\zeta^{2}}\right]_{1}^{1 / \chi^{2}}
\end{aligned}
$$

which have just been evaluated using the volume term of (17). Hence (B-1) may be rewritten as:

$$
\begin{aligned}
& \Sigma_{33}=\mathcal{A}+3 \operatorname{sgn}(\bar{D}) \zeta \mathcal{B}+\operatorname{sgn}\left(D_{33}\right) \Sigma^{\text {surf }} \\
& \Sigma_{31}=\operatorname{sgn}\left(\bar{D} D_{31}\right) \sqrt{1-3 \zeta^{2}} \mathcal{B}
\end{aligned}
$$

Relations (B-3) represent parametric equations of the yield surface. Elimination of $\zeta$ leading to an explicit relationship between stress components $\Sigma_{33}$ and $\Sigma_{31}$ is somewhat tedious. Its three main steps are summarized below. 
First, the definitions of $\mathcal{A}$ and $\mathcal{B}$ are, in fact, a short-cut towards such an elimination process. Indeed, expanding (B-2) $)_{1}$ and (B-2) 2 , taking the cosh of both sides of (B-2) $)_{1}$, then simplifying leads to:

$$
\begin{aligned}
& \chi^{2} \cosh \left(\sqrt{3} \frac{\mathcal{A}}{\bar{\sigma}}\right)=\sqrt{1+\zeta^{2}} \sqrt{\chi^{4}+\zeta^{2}}-\zeta^{2} \\
& \sqrt{3} \frac{|\mathcal{B}|}{\bar{\sigma}}=\sqrt{1+\zeta^{2}}-\sqrt{\chi^{4}+\zeta^{2}}
\end{aligned}
$$

Taking the square in (B-4) $)_{2}$ then permits to eliminate $\zeta$; the following relationship is then obtained in terms of the auxiliary variables $\mathcal{A}$ and $\mathcal{B}:{ }^{4}$

$$
\left(\sqrt{3} \frac{\mathcal{B}}{\bar{\sigma}}\right)^{2}+2 \chi^{2} \cosh \left(\sqrt{3} \frac{\mathcal{A}}{\bar{\sigma}}\right)-\left(1+\chi^{4}\right)=0
$$

Second, to obtain the explicit yield criterion, the quantities $\mathcal{A}$ and $\mathcal{B}$ need to be replaced with $\Sigma_{33}$ and $\Sigma_{31}$ in (B-5). A useful intermediate result obtained from (B-4) is

$$
1-\chi^{2} \cosh \left(\sqrt{3} \frac{\mathcal{A}}{\bar{\sigma}}\right)=1+\zeta^{2}-\sqrt{1+\zeta^{2}} \sqrt{\chi^{4}+\zeta^{2}}=\sqrt{3} \frac{|\mathcal{B}|}{\bar{\sigma}} \sqrt{1+\zeta^{2}}
$$

Taking the squares of (B-3) 2 and (B-6), one gets

$$
\left(\frac{\Sigma_{31}}{\bar{\sigma}}\right)^{2}=4\left(\frac{\mathcal{B}}{\bar{\sigma}}\right)^{2}-\left[1-\chi^{2} \cosh \left(\sqrt{3} \frac{\mathcal{A}}{\bar{\sigma}}\right)\right]^{2}
$$

where $\zeta$ is no longer present. Using then equation (B-5) one obtains:

$$
\left(\frac{\Sigma_{31}}{\bar{\sigma}}\right)^{2}=4\left(\frac{\mathcal{B}}{\bar{\sigma}}\right)^{2}-\frac{1}{4}\left[1-\chi^{4}+\left(\sqrt{3} \frac{\mathcal{B}}{\bar{\sigma}}\right)^{2}\right]^{2},
$$

which is a quadratic equation in $(\mathcal{B} / \bar{\sigma})^{2}$. Discarding the largest root by noting that $(\sqrt{3} \mathcal{B} / \bar{\sigma})^{2} \leq\left(1-\chi^{2}\right)^{2}<$ 1 from $(B-5)$, we retain the solution:

$$
\left(\sqrt{3} \frac{\mathcal{B}}{\bar{\sigma}}\right)^{2}=\frac{5}{3}+\chi^{4}-\frac{2}{3} \sqrt{4+12 \chi^{4}-3\left(\sqrt{3} \frac{\Sigma_{31}}{\bar{\sigma}}\right)^{2}}
$$

where the $\sqrt{3}$ factors are introduced to evidence the yield stress in shear $\bar{\tau} \equiv \bar{\sigma} / \sqrt{3}$.

Third, to obtain $\mathcal{A}$ note that by (B-3) $)_{2}$,

$$
(\zeta \mathcal{B})^{2}=\frac{1}{3}\left(\mathcal{B}^{2}-\Sigma_{31}^{2}\right)
$$

Hence (B-3) 1 yields:

$$
\mathcal{A}=\Sigma_{33}-\operatorname{sgn}\left(D_{33}\right) \Sigma^{\mathrm{surf}}-\operatorname{sgn}(\zeta \mathcal{B} \bar{D}) \sqrt{3\left(\mathcal{B}^{2}-\Sigma_{31}^{2}\right)}
$$

Now $\operatorname{sgn}(\zeta)=\operatorname{sgn}\left(D_{33}\right)=\operatorname{sgn}(\bar{D})$ by definition; hence $\operatorname{sgn}(\zeta \mathcal{B} \bar{D})=\operatorname{sgn}(\mathcal{B})$. Furthermore it follows from $(\mathrm{B}-2)_{2}$ that $\operatorname{sgn}(\mathcal{B})=\operatorname{sgn}(\bar{D})=\operatorname{sgn}\left(D_{33}\right)$. Finally $\operatorname{sgn}\left(D_{33}\right)=\operatorname{sgn}\left(\Sigma_{33}\right)$ since all three terms in the right-hand side of (B-3) 1 have the sign of $\bar{D}$ or $D_{33}$. Hence (B-11) may be rewritten as

$$
\begin{aligned}
\mathcal{A} & =\Sigma_{33}-\operatorname{sgn}\left(\Sigma_{33}\right) \Sigma^{\text {surf }}-\operatorname{sgn}\left(\Sigma_{33}\right) \sqrt{3\left(\mathcal{B}^{2}-\Sigma_{31}^{2}\right)} \\
& =\operatorname{sgn}\left(\Sigma_{33}\right)\left[\left|\Sigma_{33}\right|-\Sigma^{\text {surf }}-\sqrt{3\left(\mathcal{B}^{2}-\Sigma_{31}^{2}\right)}\right]
\end{aligned}
$$

\footnotetext{
${ }^{4}$ These steps are similar to those followed in a "modern" derivation of the Gurson model, e.g. Benzerga and Leblond (2010).
} 
Using (B-5), one thus gets the equation of the regular parts of the yield surface in the form:

$$
\Phi=\left(\sqrt{3} \frac{\mathcal{B}}{\bar{\sigma}}\right)^{2}+2 \chi^{2} \cosh \left[\sqrt{3}\left(\frac{\left|\Sigma_{33}\right|-\Sigma^{\text {surf }}}{\bar{\sigma}}-\sqrt{\left(\sqrt{3} \frac{\mathcal{B}}{\bar{\sigma}}\right)^{2}-\left(\sqrt{3} \frac{\Sigma_{31}}{\bar{\sigma}}\right)^{2}}\right)\right]-\left(1+\chi^{4}\right)=0
$$

where the quantity $\mathcal{B}^{2}$ is related to $\Sigma_{31}$ by (B-9).

In the limit $\chi \rightarrow 1, \Sigma_{33}=\Sigma_{31}=0$; indeed $\mathcal{A}$ and $\mathcal{B}$ must be zero by (B-5); then $\Sigma_{31}=0$ by (B-9), so that by (B-12), $\Sigma_{33}=\operatorname{sgn}\left(\Sigma_{33}\right) \Sigma^{\operatorname{surf}}=0$ by (10). Also, it can be checked that the points $\left(\Sigma_{31}= \pm \mathcal{T}, \Sigma_{33}= \pm \Sigma^{\text {surf }}\right)$, which lie at the intersections of the straight singular parts and curved regular parts, do satisfy criterion (B-13). Indeed, in such cases $\sqrt{3}|\mathcal{B}| / \bar{\sigma}=\sqrt{3}\left|\Sigma_{31}\right| / \bar{\sigma}=1-\chi^{2}$.

\section{Appendix C Quasi-Periodic Boundary Conditions on the Unit Cell}

The boundary conditions imposed on a quarter of the unit cell are expounded here. The normal and tangential displacements are the two independent degrees of freedom at each center-line. Fig. C-1 shows the various surfaces and edges of the computational domain, together with an example mesh as well as the nomenclature used.

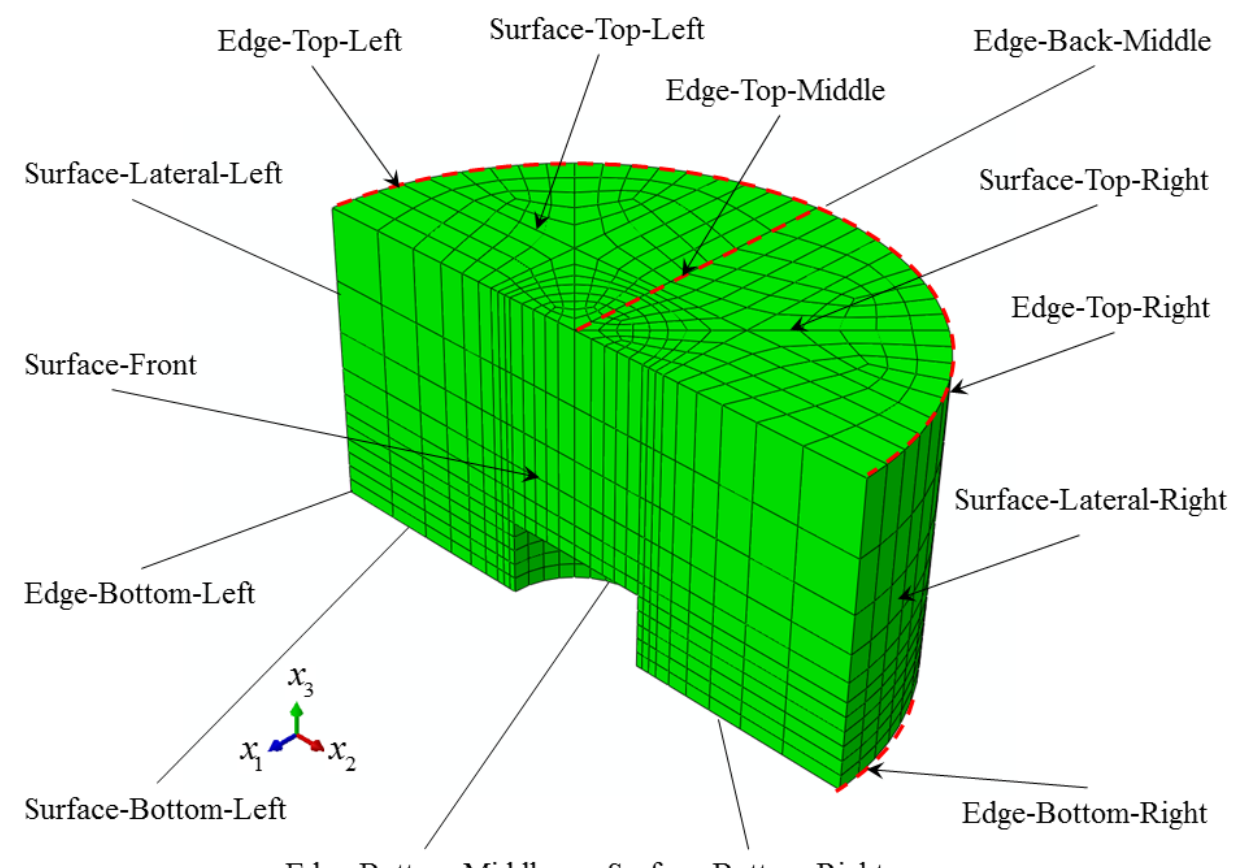

Edge-Bottom-Middle

Surface-Bottom-Right

Figure C-1: Finite element mesh for one quarter of an example unit cell with microstructural parameters given as $(\chi, W)=(0.25,1.0)$, accompanied by the nomenclature used to define various surfaces and edges to which the boundary conditions are imposed. The origin of the reference coordinate system stands at the void center in the undeformed configuration. 
Edge-Top-Middle

$$
\begin{aligned}
u_{2}\left(x_{1}, 0, H\right) & =\frac{1}{2} U_{t}, \\
u_{3}\left(x_{1}, 0, H\right) & =\frac{1}{2} U_{3} .
\end{aligned}
$$

Surface-Top-Left/Surface-Top-Right

$$
\begin{aligned}
& u_{1}\left(x_{1},-x_{2}, H\right)-u_{1}\left(x_{1}, x_{2}, H\right)=0, \\
& u_{2}\left(x_{1},-x_{2}, H\right)+u_{2}\left(x_{1}, x_{2}, H\right)=U_{t}, \\
& u_{3}\left(x_{1},-x_{2}, H\right)+u_{3}\left(x_{1}, x_{2}, H\right)=U_{3} .
\end{aligned}
$$

Edge-Top-Left/Edge-Top-Right

$$
\begin{aligned}
& u_{1}\left(x_{1},-\sqrt{L^{2}-x_{1}^{2}}, H\right)=u_{1}\left(x_{1}, \sqrt{L^{2}-x_{1}^{2}}, H\right)=\frac{x_{1}}{2 L} U_{1}, \\
& u_{2}\left(x_{1}, \pm \sqrt{L^{2}-x_{1}^{2}}, H\right)=\frac{1}{2}\left(U_{t} \pm \frac{ \pm \sqrt{L^{2}-x_{1}^{2}}}{L} U_{2}\right), \\
& u_{3}\left(x_{1}, \pm \sqrt{L^{2}-x_{1}^{2}}, H\right)=\frac{1}{2} U_{3} .
\end{aligned}
$$

Surface-Lateral-Left/Surface-Lateral-Right

$$
\begin{aligned}
& u_{1}\left(x_{1},-\sqrt{L^{2}-x_{1}^{2}}, x_{3}\right)=u_{1}\left(x_{1}, \sqrt{L^{2}-x_{1}^{2}}, x_{3}\right)=\frac{x_{1}}{2 L} U_{1}, \\
& u_{2}\left(x_{1},-\sqrt{L^{2}-x_{1}^{2}}, x_{3}\right)-u_{2}\left(x_{1}, \sqrt{L^{2}-x_{1}^{2}}, x_{3}\right)=-\frac{\sqrt{L^{2}-x_{1}^{2}}}{L} U_{2}, \\
& u_{3}\left(x_{1},-\sqrt{L^{2}-x_{1}^{2}}, x_{3}\right)-u_{3}\left(x_{1}, \sqrt{L^{2}-x_{1}^{2}}, x_{3}\right)=0 .
\end{aligned}
$$

Edge-Bottom-Left/Edge-Bottom-Right

$$
\begin{aligned}
& u_{1}\left(x_{1},-\sqrt{L^{2}-x_{1}^{2}}, 0\right)=u_{1}\left(x_{1}, \sqrt{L^{2}-x_{1}^{2}}, 0\right)=\frac{x_{1}}{2 L} U_{1}, \\
& u_{2}\left(x_{1}, \pm \sqrt{L^{2}-x_{1}^{2}}, 0\right)= \pm \frac{\sqrt{L^{2}-x_{1}^{2}}}{2 L} U_{2} \\
& u_{3}\left(x_{1}, \pm \sqrt{L^{2}-x_{1}^{2}}, 0\right)=0 .
\end{aligned}
$$

Edge-Bottom-Middle

$$
\begin{aligned}
& u_{2}\left(x_{1}, 0,0\right)=0 \\
& u_{3}\left(x_{1}, 0,0\right)=0
\end{aligned}
$$

Surface-Bottom-Left/Surface-Bottom-Right

$$
\begin{aligned}
& u_{1}\left(x_{1},-x_{2}, 0\right)-u_{1}\left(x_{1}, x_{2}, 0\right)=0, \\
& u_{2}\left(x_{1},-x_{2}, 0\right)+u_{2}\left(x_{1}, x_{2}, 0\right)=0, \\
& u_{3}\left(x_{1},-x_{2}, 0\right)+u_{3}\left(x_{1}, x_{2}, 0\right)=0 .
\end{aligned}
$$


Edge-Back-Middle

$$
u_{1}\left(-L, 0, x_{3}\right)=-\frac{1}{2} U_{1}
$$

Surface-Front

$$
u_{1}\left(0, x_{2}, x_{3}\right)=0
$$

\section{Appendix D Rationale for Heuristic Modification}

Criterion (19) $)_{1}$ is modified as follows:

$$
\frac{\mathcal{B}^{2}}{b \bar{\tau}^{2}}+2 f_{\mathrm{b}} \cosh \left[\frac{\left|\Sigma_{33}\right|-t \Sigma^{\text {surf }}}{\bar{\tau}}-\sqrt{3 \frac{\mathcal{B}^{2}-\left(\Sigma_{31} / l\right)^{2}}{\bar{\tau}^{2}}}\right]-\left(1+f_{\mathrm{b}}^{2}\right)=0
$$

where $t, b$ and $l$ are parameters. It is unlikely that constant parameters will fit all purposes. Therefore, $t, b$ and $l$ are considered a priori functions of the internal parameters $\chi$ and $W$. The idea is to obtain the simplest functions that reduce the error between the numerical results and model predictions shown in Fig. 6.

Firstly, parameters $t$ and $b$ are introduced in such a way that the same heuristic modification of Torki et al. (2015) is arrived at. There it was shown that a constant value of $b \sim 1$ is appropriate. Also, a homographic function of $W$ was necessary for $t$ in order to remove the singular behavior for $W \rightarrow 0$. This behavior manifests in the absence of any shear. The criterion in this case reduces to:

$$
\left.\frac{\Sigma_{33}}{\bar{\sigma}}\right|_{\Sigma_{31}=0}=t \Sigma^{\mathrm{surf}}+b \Sigma^{\mathrm{vol}}
$$

following the same procedure as for obtaining (A-8). The simplest choice for function $t$ is:

$$
t(\chi, W)=\frac{\left(t_{0}+t_{1} \chi\right) W}{1+\left(t_{0}+t_{1} \chi\right) W}
$$

where $t_{0}$ and $t_{1}$ are parameters to be determined on the basis of the numerical results. In doing so, we improve upon the proposal of Torki et al. (2015) by limiting the heuristic modification to the range $\chi \geq 0.2$ and taking $t(\chi, W)=t(0.2, W)$ for $\chi<0.2$ so that the exact limit $\Sigma_{33} \rightarrow \infty$ is retained for $\chi \rightarrow 0$. The choice of parameters $t_{0}=-1.3, t_{1}=20.6$ and $b=1.0$ proves quite good.

Secondly, parameter $l$ aims at reducing the error in the case of prolate voids under shear-dominated loadings. Indeed, the modified criterion (D-1) reduces to $\Sigma_{31}=l(W, \chi) \mathcal{T}$ in pure shear. The fact that $l$ should depend on $W$ is easily inferred from the computational results. The simplest possible form for $l(W, \chi)$ is a bi-linear function (again for $\chi \geq 0.2$ only):

$$
l(\chi, W)=\left[1+\left(l_{1} \chi+l_{0}\right) W\right] \mathcal{T}
$$

The results shown in Fig. 6 were obtained using the choice $\left(l_{0}, l_{1}\right)=(0.035,-0.15)$. 


\section{References}

I. Barsoum and J. Faleskog. Rupture mechanisms in combined tension and shear-Experiments. International Journal of Solids and Structures, 44:1768-1786, 2007 a.

I. Barsoum and J. Faleskog. Rupture mechanisms in combined tension and shear-Micromechanics. International Journal of Solids and Structures, 44:5481-5498, $2007 \mathrm{~b}$.

I. Barsoum and J. Faleskog. Micromechanical analysis on the influence of the Lode parameter on void growth and coalescence. International Journal of Solids and Structures, 48:925-938, 2011.

A. A. Benzerga. Rupture ductile des tôles anisotropes. PhD thesis, Ecole Nationale Supérieure des Mines de Paris, 2000.

A. A. Benzerga. Micromechanics of Coalescence in Ductile Fracture. Journal of the Mechanics and Physics of Solids, 50:1331-1362, 2002.

A. A. Benzerga and J. Besson. Plastic potentials for anisotropic porous solids. European Journal of Mechanics, 20A:397-434, 2001.

A. A. Benzerga and J.-B. Leblond. Ductile fracture by void growth to coalescence. Advances in Applied Mechanics, 44:169-305, 2010.

A. A. Benzerga and J.-B. Leblond. Effective Yield Criterion Accounting for Microvoid Coalescence. Journal of Applied Mechanics, 81:031009, 2014.

A. A. Benzerga, J. Besson, and A. Pineau. Coalescence-Controlled Anisotropic Ductile Fracture. Journal of Engineering Materials and Technology, 121:221-229, 1999.

A. A. Benzerga, J. Besson, R. Batisse, and A. Pineau. Synergistic effects of plastic anisotropy and void coalescence on fracture mode in plane strain. Modelling and Simulation in Materials Science and Engineering, 10:73-102, 2002.

A. A. Benzerga, J. Besson, and A. Pineau. Anisotropic ductile fracture. Part II: theory. Acta Materialia, 52: 4639-4650, 2004.

A. A. Benzerga, J.-B. Leblond, A. Needleman, and V. Tvergaard. Ductile Failure Modeling. International Journal of Fracture, 201:29-80, 2016.

K. Danas and P. Ponte Castañeda. Influence of the Lode parameter and the stress triaxiality on the failure of elasto-plastic porous materials. International Journal of Solids and Structures, 49:1325-1342, 2012.

M. Dunand and D. Mohr. Optimized butterfly specimen for the fracture testing of sheet materials under combined normal and shear loading. Engineering Fracture Mechanics, 78:2919-2934, 2011.

M. Dunand and D. Mohr. Effect of Lode parameter on plastic flow localization after proportional loading at low stress triaxialities. Journal of the Mechanics and Physics of Solids, 66:133-153, 2014.

F. Fritzen, S. Forest, T. Böhlke, D. Kondo, and T. Kanit. Computational homogenization of elasto-plastic porous metals. International Journal of Plasticity, 29:102-119, 2012.

M. Gologanu, J.-B. Leblond, G. Perrin, and J. Devaux. Theoretical models for void coalescence in porous ductile solids - I: Coalescence in "layers". International Journal of Solids and Structures, 38:5581-5594, 2001. 
A. L. Gurson. Continuum Theory of Ductile Rupture by Void Nucleation and Growth: Part I- Yield Criteria and Flow Rules for Porous Ductile Media. Journal of Engineering Materials and Technology, 99:2-15, 1977.

J. Hure and P. O. Barrioz. Theoretical estimates for flat voids coalescence by internal necking. European Journal of Mechanics, 60:217-226, 2016.

S. M. Keralavarma and A. A. Benzerga. A constitutive model for plastically anisotropic solids with nonspherical voids. Journal of the Mechanics and Physics of Solids, 58:874-901, 2010.

S. M. Keralavarma and S. Chockalingam. A Criterion for Void Coalescence in Anisotropic Ductile Materials. International Journal of Plasticity, 82:159-176, 2016.

J. Koplik and A. Needleman. Void growth and coalescence in porous plastic solids. International Journal of Solids and Structures, 24(8):835-853, 1988.

J.-B. Leblond and G. Mottet. A theoretical approach of strain localization within thin planar bands in porous ductile materials. Comptes Rendus Mecanique, 336:176-189, 2008.

Z. G. Liu, W. H. Wong, and T. F. Guo. Void behaviors from low to high triaxialities: Transition from void collapse to void coalescence. International Journal of Plasticity, 84:183-202, 2016.

K. Madou and J.-B. Leblond. A Gurson-type criterion for porous ductile solids containing arbitrary ellipsoidal voids - I: Limit-analysis of some representative cell. Journal of the Mechanics and Physics of Solids, 60:1020-1036, 2012.

K. Madou and J.-B. Leblond. Numerical studies of porous ductile materials containing arbitrary ellipsoidal voids - I: Yield surfaces of representative cells. European Journal of Mechanics, 42:480-489, 2013.

L. Morin, J.-B. Leblond, and A. A. Benzerga. Coalescence of voids by internal necking: theoretical estimates and numerical results. Journal of the Mechanics and Physics of Solids, 75:140-158, 2015.

L. Morin, J.-B. Leblond, A. A. Benzerga, and D. Kondo. A unified criterion for the growth and coalescence of microvoids. Journal of the Mechanics and Physics of Solids, 97:19-36, 2016.

K. L. Nielsen, J. Dahl, and V. Tvergaard. Collapse and coalescence of spherical voids subject to intense shearing: studied in full 3D. International Journal of Fracture, 177:97-108, 2012.

T. Pardoen and J. W. Hutchinson. An extended model for void growth and coalescence. Journal of the Mechanics and Physics of Solids, 48:2467-2512, 2000.

A. Pineau, A. A. Benzerga, and T. Pardoen. Failure of metals I. Brittle and Ductile Fracture. Acta Materialia, 107:424-483, 2016.

J.R. Rice. The localization of plastic deformation. In W.T. Koiter, editor, 14th int. cong. Theoretical and Applied Mechanics, pages 207-220. North-Holland, Amsterdam, 1976.

F. Scheyvaerts, P. R. Onck, C. Tekog̃lu, and T. Pardoen. The growth and coalescence of ellipsoidal voids in plane strain under combined shear and tension. Journal of the Mechanics and Physics of Solids, 59: 373-397, 2011.

C. Tekog̃lu. Representative volume element calculations under constant stress triaxiality, Lode parameter, and shear ratio. International Journal of Solids and Structures, 51:4544-4553, 2014. 
C. Tekog̃lu, J.-B. Leblond, and T. Pardoen. A criterion for the onset of void coalescence under combined tension and shear. Journal of the Mechanics and Physics of Solids, 60:1363-1381, 2012.

P. F. Thomason. Three-dimensional models for the plastic limit-loads at incipient failure of the intervoid matrix in ductile porous solids. Acta Metallurgica, 33:1079-1085, 1985.

M. E. Torki, A. A. Benzerga, and J.-B. Leblond. On Void Coalescence under Combined Tension and Shear. Journal of Applied Mechanics, 82(7):071005, 2015.

V. Tvergaard. Effect of stress-state and spacing on voids in a shear-field. International Journal of Solids and Structures, 49:3047-3054, 2012. 